International Journal of Modern Physics B,

(C) World Scientific Publishing Company

\title{
Quantum Effects and Broken Symmetries in Frustrated Antiferromagnets
}

\author{
LUCA CAPRIOTTI \\ Istituto Nazionale per la Fisica della Materia (INFM), Unità di Firenze, \\ Largo E. Fermi 2, I-50125 Firenze, Italy
}

Received (received date)

Revised (revised date)

\begin{abstract}
We investigate the interplay between frustration and zero-point quantum fluctuations in the ground state of the triangular and $J_{1}-J_{2}$ Heisenberg antiferromagnets, using finitesize spin-wave theory, exact diagonalization, and quantum Monte Carlo methods. In the triangular Heisenberg antiferromagnet, by performing a systematic size-scaling analysis, we have obtained strong evidences for a gapless spectrum and a finite value of the thermodynamic order parameter, thus confirming the existence of long-range Néel order. The good agreement between the finite-size spin-wave results and the exact and quantum Monte Carlo data also supports the reliability of the spin-wave expansion to describe both the ground state and the low-energy spin excitations of the triangular Heisenberg antiferromagnet. In the $J_{1}-J_{2}$ Heisenberg model, our results indicate the opening of a finite gap in the thermodynamic excitation spectrum at $J_{2} / J_{1} \simeq 0.4$, marking the melting of the antiferromagnetic Néel order and the onset of a non-magnetic ground state. In order to characterize the nature of the latter quantum-disordered phase we have computed the susceptibilities for the most important crystal symmetry breaking operators. In the ordered phase the effectiveness of the spin-wave theory in reproducing the low-energy excitation spectrum suggests that the uniform spin susceptibility of the model is very close to the linear spin-wave prediction.
\end{abstract}

Keywords: The contents of the keywords

\section{Introduction}

The physics of quantum antiferromagnets is a very old topic, dating back to the early days of quantum mechanics itself. Nonetheless, after many years of intensive study, the interest in this research field is still high, with several new problems arising from the behavior of low-dimensional magnetic materials. This is also due to the existence of simple toy-models in which the interplay between antiferromagnetism, symmetry, dimensionality and strong quantum correlations leads to fascinating effects in the low-temperature physics, often reproducing the behavior of real systems. Among them, the nearest-neighbor Heisenberg Hamiltonian,

$$
\hat{\mathcal{H}}=J \sum_{n . n .} \hat{\mathbf{S}}_{i} \cdot \hat{\mathbf{S}}_{j}
$$

where $\hat{\mathbf{S}}_{i}=\left(\hat{S}_{i}^{x}, \hat{S}_{i}^{y}, \hat{S}_{i}^{z}\right)$ are spin- $s$ operators and $J$ is the (positive) exchange inte- 
gral, has certainly played a central role as an ideal test ground to investigate the influence of quantum effects on the mechanism of spontaneous symmetry breaking. In fact, in contrast to the ferromagnetic state, the classical Néel state is not an eigenstate of the Heisenberg Hamiltonian and therefore, in general, the ground state of the latter does not have a purely classical representation. Hence, quantum effects may play an important role in modifying the zero-temperature properties of the model from the classical $(s \rightarrow \infty)$ limit. In particular, reduced dimensionality and a small spin value might enhance zero-point quantum fluctuations up to the point of destroying the classical Néel order, thus stabilizing a ground state with symmetries and correlations different from its classical counterpart.

Indeed, in one dimension and for $s=1 / 2$, a famous exact solution found by Bethe in 1931 (Ref. 4 ) showed that quantum effects prevent the onset of true longrange antiferromagnetic order, giving instead a power-law decay of the spin-spin correlation functions. Despite Bethe's promise to generalize his solution to the two-dimensional square lattice case, appearing in the conclusions of his paper, this was never done, and the issue of the existence of long-range order in the ground state of the two-dimensional Heisenberg model has been left unsolved for many years. The rigorous proof of the ordered nature of the ground state of the square Heisenberg antiferromagnet was given in fact, for $s \geq 1$, only in 1986 3 and has not been extended yet to the spin-half case where zero-point quantum fluctuations are stronger.

This problem became a hot topic when possible connections between a nonmagnetic ground state and the mechanism of high- $T_{c}$ superconductivity were put forward by Anderson in 1987.3 In fact, since the stoichiometric compounds of the high- $T_{c}$ superconductors are good realizations of a $s=1 / 2$ square Heisenberg antiferromagnet, this conjecture focused the attention on the properties of this system. Fortunately enough, at that time the development of modern computers was such that the use of numerical techniques could compensate for the lack of exact analytical results. In particular, quantum Monte Carlo methods have been of crucial importance, by allowing one to perform a systematic size-scaling of the physical observables and therefore to reach a definite conclusion. As a result, even if a rigorous proof is still lacking, there is at present a general consensus about the ordered nature of the ground state of the spin-half square Heisenberg antiferromagnet: in two dimensions, reduced dimensionality and a low spin value do not seem enough to stabilize, within the Heisenberg model, a non-magnetic ground state.

Better candidates for a realization of disordered ground states in two dimensions are frustrated spin models. In these systems, in fact, the usual antiferromagnetic alignment between spins is hindered by the geometry of the lattice or by the presence of competing interactions. As a result, a general feature introduced by frustration is a less stable classical minimum energy configuration which is more likely to be destabilized by zero-point quantum fluctuations for a small spin value. Among this class of systems two prototypical examples are given by the triangular Heisenberg antiferromagnet, and the $J_{1}-J_{2}$ Heisenberg model. The nature of the ground state 


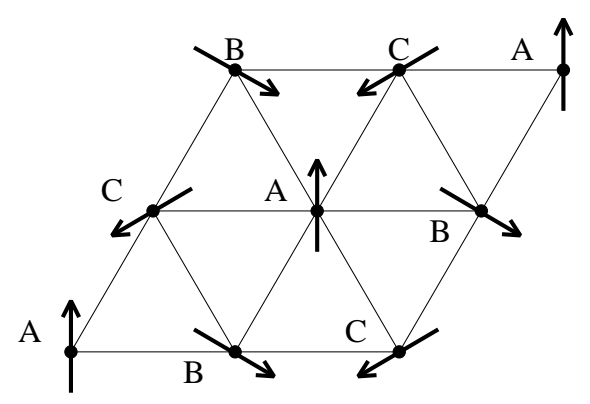

Figure 1: The classical Néel state consists of coplanar spins forming $\pm 2 \pi / 3$ angles between nearest neighbors. This leads to a $\sqrt{3} \times \sqrt{3}$ periodicity with the spins on the three sublattices A,B,C ferromagnetically aligned.

in these frustrated spin models represents the main topic of this paper.

The triangular Heisenberg antiferromagnet is described by the Hamiltonian (11), where $i$ and $j$ are the sites of a triangular lattice. Due to the geometry of the lattice (see Fig. 1), the classical minimum energy configuration of this model is not the usual Néel state with antiparallel spins on neighboring sites. In fact, if two spins on an elementary triangular plaquette minimize their exchange energy by aligning antiparallel, the third one cannot do the same because it cannot be antiparallel to both of them, simultaneously. As a result, the minimum energy configuration consists of coplanar spins forming $\pm 2 \pi / 3$ angles between nearest-neighbors and this leads to a $\sqrt{3} \times \sqrt{3}$ periodic Néel state with the spins ferromagnetically aligned on each of the three sublattices (Fig. 1). The resulting state, having an energy per bond twice than the optimal one, is far less stable than that on the square lattice.

In the $J_{1}-J_{2}$ model, instead, frustration arises on the square lattice because of the presence of competing interactions, the Hamiltonian being

$$
\hat{\mathcal{H}}=J_{1} \sum_{n . n .} \hat{\mathbf{S}}_{i} \cdot \hat{\mathbf{S}}_{j}+J_{2} \sum_{n . n . n .} \hat{\mathbf{S}}_{i} \cdot \hat{\mathbf{S}}_{j}
$$

where $J_{1}$ and $J_{2}$ are the antiferromagnetic couplings between nearest- and nextnearest-neighbors, respectively. Classically, the minimum energy configuration has the conventional Néel order for $J_{2} / J_{1}<0.5$ [Fig. 2 (a)]. By increasing further the frustrating interaction $J_{2}$ this configuration is destabilized and, for $J_{2} / J_{1}>0.5$, the system decouples into two Néel ordered sublattices. At the purely classical level, the energy of the latter configuration is independent of the relative orientations of the staggered magnetizations on the two sublattices. However, this degeneracy is partially lifted by zero-point quantum fluctuations even at the lowest order in $1 / s$ so that in the $s \rightarrow \infty$ limit the minimum energy configuration is the so-called collinear state [Fig. 2(b)] with the spin ferromagnetically aligned in one direction and antiferromagnetically in the other, corresponding to a magnetic wavevector $\mathbf{Q}=(\pi, 0)$ or $\mathbf{Q}=(0, \pi) .5$ Exactly at $J_{2} / J_{1}=0.5$ any classical state having zero total spin on each elementary square plaquette is a minimum of the total energy. 


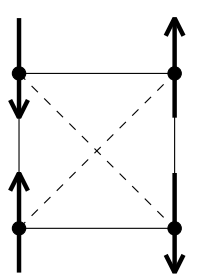

(a)

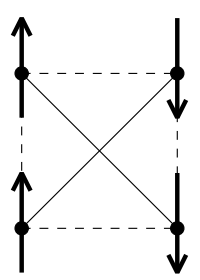

(b)

Figure 2: The two sublattices Néel (a) and the collinear (b) classical states.

These states include both the Néel and the collinear states but also many others with no long-range order. The occurrence of a non-magnetic ground state in the quantum case, for a small spin value, is therefore likely around this value of the $J_{2} / J_{1}$ ratio.

The recent experimental finding of real compounds described by the triangular and the $J_{1}-J_{2}$ Heisenberg antiferromagnets have renewed the interest in these frustrated spin systems. In particular, the $\mathrm{K} / \mathrm{Si}(111)-\sqrt{3} \times \sqrt{3}$-B interface $\mathrm{Q}$, has turned out to be a good experimental realization of a spin-half Heisenberg antiferromagnet on a triangular lattice. In fact, due to strong electronic correlations, the surfaces states consist of a triangular arrangement of half-filled dangling bonds, which are localized and carry local $s=1 / 2$ magnetic moments coupled antiferromagnetically. Recent experimental realizations of the spin-half $J_{1}-J_{2}$ Heisenberg model have been found instead in the $\mathrm{Li}_{2} \mathrm{VOSiO}_{4}$ and $\mathrm{Li}_{2} \mathrm{VOGeO}_{4}$ compounds. These are threedimensional systems formed by stacked square planes of $V^{4+}(s=1 / 2)$ ions with a weak inter-plane interaction. The structure of the $V^{4+}$ planes suggests that both the superexchange couplings between first and second neighbors can be significant and indeed, the first experimental results have indicated that these antiferromagnetic couplings are of the same order of magnitude. $\mathrm{B}$ In addition, the possibility of performing measurements under pressure will also allow one in the near future 9 to tune the $J_{2} / J_{1}$ ratio and to investigate the properties of these systems in various regimes of frustration.

In this work the problem of the nature of the ground state of these frustrated spin systems is tackled using various techniques, namely: the finite-size spin-wave theory, exact diagonalization of small clusters by the Lanczos algorithm, and several zero-temperature quantum Monte Carlo methods. The finite-size spin-wave theory has been recently proposed 10 as a generalization of one of the oldest analytical techniques in the study of quantum magnetism.11 In particular, this spin-wave expansion allows one to deal with finite clusters while avoiding the spurious Goldstone modes divergences in a straightforward way. Even if these results are biased by the long-range order hypothesis, nevertheless useful information on the thermodynamic 
ground state can be extracted from the comparison with the numerical results on finite systems or from the occurrence of a breakdown of the $1 / \mathrm{s}$ expansion.

The Lanczos method 12 allows the exact evaluation of static and dynamical properties of the finite-size system and, especially when combined with a careful analysis of the symmetry of the low-energy excited states 13 can provide clear indications about the nature of the ground state. However, due to memory constraints, exact diagonalizations techniques are limited in two dimensions to very small clusters $(\sim 30$ sites $)$ so that it is in general difficult to perform a systematic size scaling of the important physical observables. In order to numerically investigate larger systems, different approaches are therefore necessary.

In the unfrustrated cases, quantum Monte Carlo has turned out to be an essential instrument for studying both the ground-state and the finite-temperature properties of a quantum antiferromagnet.14 Unfortunately, in the frustrated cases standard stochastic techniques cannot be applied, as their reliability is strongly limited by the well-known sign problem. This numerical instability originates from the vanishing of the signal-to-noise ratio in the Monte Carlo sampling which occurs within bosonic models in the presence of frustration or, in general, in fermionic systems.

Presently, the sign problem can be controlled only at the price of introducing some kind of approximation. Apart from purely variational calculations, the simplest approximation scheme in the framework of one of the most efficient zerotemperature algorithms - the Green function Monte Carlo 15 - is the fixed-node (FN) technique. 1 In this technique, the exact imaginary time propagator $e^{-\tau \hat{\mathcal{H}}}$ used to filter out the ground state from the best variational guess $\left|\psi_{G}\right\rangle$-is replaced by an approximate propagator $e^{-\tau \hat{\mathcal{H}}_{\mathrm{FN}}}$ such that the nodes of the propagated state $e^{-\tau \hat{\mathcal{H}}_{\mathrm{FN}}}\left|\psi_{G}\right\rangle$ do not change, due to an appropriate choice of the effective FN Hamiltonian $\hat{\mathcal{H}}_{\mathrm{FN}}$ (which in turn depends on $\left|\psi_{G}\right\rangle$ ). The FN approximation becomes exact if the so called guiding wavefunction $\left|\psi_{G}\right\rangle$ is the exact ground state. However, the fixed-node results are usually strongly biased by this ansatz so that it is in general difficult to extract reliable information about the ground-state correlations whenever they are not well reproduced by the variational guess.

In order to overcome this difficulty, here we have used the recently developed Green function Monte Carlo with Stochastic Reconfiguration (GFMCSR), 17 , to which allows one to release the fixed-node approximation in a controlled way and to obtain much more accurate estimates of the ground-state correlations, thus reproducing also ground-state properties that are not contained at the variational level in the guiding wavefunction. During each short imaginary time evolution $\tau \rightarrow \tau+\Delta \tau$, where both the exact and the approximate propagation can be performed without sign problem instabilities, the FN dynamic is systematically improved by requiring that a given number $p$ of mixed averages 17 of correlation functions are propagated consistently with the exact dynamic. By increasing the number of correlation functions one typically improves the accuracy of the calculation since the method becomes exact if all the independent correlation functions are included in the stochastic reconfiguration (SR) scheme. Typically, 19,20 few correlation functions 
$(p \sim 10)$ allow to obtain rather accurate values of the ground-state energy with an error much less than $1 \%$ - for lattice sizes $(N \leq 36)$ where the exact solution is available numerically - and without a sizable loss of accuracy with increasing size. Such accuracy is usually enough to reproduce some physical features that are not contained at the variational level.

In this paper, using these numerical techniques, after a brief review of the basic concepts of spontaneous symmetry breaking in a quantum antiferromagnet (Sec. 2), we provide two clear examples of systems in which the combined effect of frustration and quantum fluctuations do not or do change the zero-temperature long-range properties of their classical counterparts. In particular, we find that the thermodynamic ground state of the spin-half triangular Heisenberg antiferromagnet (Sec. 3) is most likely long-range ordered although with a remarkable reduction of the order parameter with respect to the classical case. On the other hand, in the $J_{1}-J_{2}$ Heisenberg model (Sec. 4), quantum fluctuations turn out to be strong enough to melt the antiferromagnetic Néel order, driving the ground state into a non-magnetic phase of purely quantum-mechanical nature.

\section{Spontaneous Symmetry Breaking in a Quantum Antiferromagnet}

In this section we will review the basic concepts concerning the mechanism of the spontaneous symmetry breaking in a quantum antiferromagnet that will be used in the present work to investigate the ground-state properties of the triangular and of the $J_{1}-J_{2}$ Heisenberg models. Starting from the Lieb-Mattis theorem for the bipartite Heisenberg antiferromagnet, we will introduce some general features of the finite-size spectrum of the Heisenberg Hamiltonian. In particular, we will focus on the importance of the structure of the low-lying excited states, explaining also how the finite-size ground-state properties can be consistent with a broken symmetry in the thermodynamic limit.

\subsection{The Lieb-Mattis property}

One of the few rigorous results on the ground-state properties of the Heisenberg model on a bipartite lattice is the Lieb-Mattis theorem. Here we will reproduce the demonstration of this important result, following the paper by E. Lieb and D. Mattis 21 who extended and generalized the original results obtained by W. Marshall.22

Let us consider the Heisenberg Hamiltonian

$$
\hat{\mathcal{H}}=\sum_{(i, j)} J_{i j} \hat{\mathbf{S}}_{i} \cdot \hat{\mathbf{S}}_{j}
$$

where the sum runs over all the bonds on a $d$-dimensional bipartite lattice, $\hat{\mathbf{S}}_{i}$ are spin- $s$ operators; $J_{i j}$ is the (symmetric) exchange matrix such that $J_{i j}>0$, if $i$ and $j$ belong to different sublattices, and $J_{i j}<0$, otherwise. We will assume that the Hamiltonian cannot be split into sets of noninteracting spins, restricting also 
for simplicity, to the case in which the number of sites of the two sublattices is the same. In the following $N$ will denote the total number of sites of the lattice.

Since the Hamiltonian (3) commutes with all the three components of the total spin operator,

$$
\hat{\mathbf{S}}=\sum_{i} \hat{\mathbf{S}}_{i}
$$

it is known from the theory of the angular momentum that we can construct two operators which commute with each other and with $\hat{\mathcal{H}}$. For example, choosing the quantization axis along the $z$-direction, we can consider the total spin squared, $\hat{\mathbf{S}}^{2}$, and its component along the $z$-axis, $\hat{S}^{z}$, whose eigenvalues $S(S+1)$ and $M$ are good quantum numbers for the eigenstates of the Hamiltonian,

$$
\left|\psi_{n}\right\rangle=|n, S, M, \cdots\rangle,
$$

such that $\hat{\mathcal{H}}\left|\psi_{n}\right\rangle=E_{n}\left|\psi_{n}\right\rangle$. If the couplings $J_{i j}$ are translationally or rotationally invariant, also the lattice momentum and the eigenvalues of the generators of the crystal point group label the eigenstates of the Hamiltonian. However this restriction is not needed to derive the following results.

\subsubsection{Marshall-Peierls sign rule}

In the hypothesis stated above, the first strong result one can prove is about the signs of the coefficients of the expansion of the ground state of (3) in the so-called Ising basis whose states are specified by assigning the value of the $S_{i}^{z}$ at each lattice site, i.e., $|x\rangle=\prod_{i=1}\left|m_{i}\right\rangle$ with $\hat{\mathbf{S}}_{i}^{2}\left|m_{i}\right\rangle=s(s+1)\left|m_{i}\right\rangle$ and $\hat{S}_{i}^{z}\left|m_{i}\right\rangle=m_{i}\left|m_{i}\right\rangle$ where $\left|m_{i}\right| \leq s$. Within this basis it is easy to distinguish between the subspaces with different values of the projection of the total spin on the $z$-axis, $M$. In fact, in order to restrict to a particular $M$ sector, one has to use only the states of the basis, $|x\rangle$, such that $\sum_{i} m_{i}=M$. In addition, sorting the basis in order to group together the states with the same $M$, the Hamiltonian matrix assumes a simpler block-diagonal form, i.e., a block for each $M$ sector. Let us restrict therefore to a particular $M$ subspace.

The first step of the proof is to perform a unitary transformation,

$$
\hat{\mathcal{U}}^{\dagger}=\exp \left[-i \pi \sum_{i \in \mathrm{B}}\left(s+\hat{S}_{i}^{z}\right)\right],
$$

whose physical meaning is to flip the quantization axis on the B sublattice. This defines a spatially varying reference frame pointing along the local Néel direction [Fig. 2 (a)]. The transformed Hamiltonian then results

$$
\hat{\mathcal{U}}^{\dagger} \hat{\mathcal{H}} \hat{\mathcal{U}}=\hat{\mathcal{H}}_{d}+\hat{\mathcal{H}}_{o}
$$

where the diagonal part,

$$
\hat{\mathcal{H}}_{d}=\sum_{(i, j)} J_{i j} \hat{S}_{i}^{z} \hat{S}_{j}^{z}
$$


is invariant in the new representation, while the off-diagonal spin-flip term,

$$
\hat{\mathcal{H}}_{o}=-\frac{1}{2} \sum_{(i, j)} J_{i j}\left(\hat{S}_{i}^{+} \hat{S}_{j}^{-}+\text {h.c. }\right),
$$

acquires instead an overall minus sign. Therefore, in this representation, the Hamiltonian has non-positive off-diagonal matrix elements. In this case, using the hypothesis that the Hamiltonian cannot be splitinto sets of noninteracting spins one can demonstrate (Perron-Frobenius theorem 23 ) that the ground-state expansion over the chosen basis $\left|\tilde{\psi}_{M}\right\rangle=\sum_{x} f_{x}|x\rangle$ has non-vanishing positive amplitudes, i.e., $f_{x}>0$.

The latter result has two important consequences: in each $M$ subspace the ground state of the Hamiltonian (3) $i$ ) is non-degenerate, and ii) obeys, in the original representation, the well-known Marshall-Peierls sign rule, i.e.,

$$
\left|\psi_{M}\right\rangle=\hat{\mathcal{U}}\left|\tilde{\psi}_{M}\right\rangle=\sum_{x} e^{i \pi N(x)} f_{x}|x\rangle=\sum_{x}(-1)^{N(x)} f_{x}|x\rangle,
$$

where $N(x)=\sum_{i \in \mathrm{B}}\left(s+m_{i}\right)$, and the sum is restricted to the configurations $|x\rangle$ such that $\sum_{i} m_{i}=M$. Notice that the unessential constant term $s$ in the definition of $\hat{\mathcal{U}}$ have allowed us to write a real ground-state wavefunction. In particular, for $s=1 / 2$, $N(x)$ is simply the number of spins up on the B sublattice, say $N_{\uparrow}(x)$. In this case, the ground-state projections on two Ising configurations differing for a single spin flip have opposite signs. Notice that the rotational invariance of the Hamiltonian has never been used in the proof so that this result is valid in general even in presence of an easy-plane anisotropy, i.e., for the more general XXZ Hamiltonian, or in presence of an arbitrary magnetic field in the $z$ direction.

In contrast, for frustrated spin systems, like the $J_{1}-J_{2}$ model and the Heisenberg triangular antiferromagnet, the same proof does not hold. In fact, in both systems the off-diagonal part of the Hamiltonian cannot be made non-positive defined by any known unitary transformation. In the $J_{1}-J_{2}$ model, the unitary transformation (6) does not change the signs of the next-nearest-neighbors spin-flip term. In the triangular antiferromagnet, under the transformation corresponding to the one in Eq. (6)

$$
\hat{\mathcal{U}}^{\dagger}=\exp \left[-\frac{2 \pi i}{3}\left(\sum_{i \in \mathrm{B}} \hat{S}_{i}^{z}-\sum_{i \in \mathrm{C}} \hat{S}_{i}^{z}\right)\right]
$$

where $\mathrm{B}$ and $\mathrm{C}$ label two of the three sublattices as shown in Fig. 1 ${ }^{a}$, the Hamiltonian reads

$$
\begin{aligned}
\hat{\mathcal{U}}^{\dagger} \hat{\mathcal{H}} \hat{\mathcal{U}} & =-\frac{J}{4} \sum_{\langle i, j\rangle}\left(\hat{S}_{i}^{+} \hat{S}_{j}^{-}+\text {h.c. }\right)+J \sum_{\langle i, j\rangle} \hat{S}_{i}^{z} \hat{S}_{j}^{z} \\
& +i \sum_{\langle i, j\rangle} C_{i, j}\left(\hat{S}_{i}^{+} \hat{S}_{j}^{-}-\text {h.c. }\right)
\end{aligned}
$$

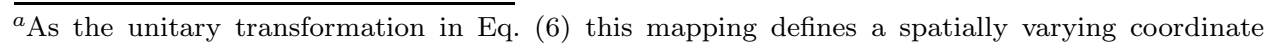
system pointing along the local Néel direction. 
Table 1. Weight of the states satisfying the Marshall-Peierls sign-rule, $\langle s\rangle$, in the ground state of the $J_{1}-J_{2}$ Heisenberg model. Data are reported for $s=1 / 2, N=32$ and $N=36$, and various values of the frustration.

\begin{tabular}{cccccc}
\hline$J_{2} / J_{1}$ & 0.1 & 0.2 & 0.3 & 0.4 & 0.5 \\
\hline$N=32$ & 1 & 1 & $\sim\left(1-10^{-8}\right)$ & $\sim 0.9998$ & 0.973 \\
$N=36$ & 1 & 1 & $\sim\left(1-10^{-8}\right)$ & $\sim 0.9995$ & 0.961 \\
\hline
\end{tabular}

with $C_{i, j}= \pm \sqrt{3} / 4$. The transformed Hamiltonian then displays an extra currentlike term which is off-diagonal and has no definite sign. Therefore for frustrated spin systems the Marshall-Peierls sign rule cannot be demonstrated and in general it does not hold exactly. In addition, the impossibility to find a unitary transformation allowing us to map the Hamiltonian into an operator with non-positive defined offdiagonal matrix elements has also dramatic consequences on the computability of such frustrated systems with standard quantum Monte Carlo methods. This is the origin of the so-called sign-problem instability 17.18

However, as originally pointed out by Richter and co-workers,24 the MarshallPeierls sign-rule survives in the $J_{1}-J_{2}$ model in very good approximation up to relatively large values of the frustration. By means of the Lanczos exact diagonalization technique, for $s=1 / 2, N=32$ and 36 , we have calculated the weight of the states satisfying the Marshall-Peierls sign-rule in the expansion of the (normalized) exact ground state $\left|\psi_{0}\right\rangle$, namely:

$$
\langle s\rangle=\sum_{x}\left|\psi_{0}(x)\right|^{2}(-1)^{N_{\uparrow}(x)} \operatorname{sgn}\left[\psi_{0}(x)\right],
$$

with $\psi(x)=\langle x \mid \psi\rangle$ and the notations introduced in this section Our results, shown in Tab. 1, put further evidence to the previous findings of Ref. 24 and indicate that the Marshall-Peierls sign rule is verified almost exactly up to $J_{2} / J_{1} \simeq 0.3 \div 0.4$, even for $N=36$. Moreover, even if the average sign $\langle s\rangle$ eventually vanishes in the thermodynamic limit, its small size dependence suggests that this property is likely to be conserved also for the lattice sizes $(N \simeq 100)$ presently accessible with the stochastic numerical techniques used in this work. A reasonable guess on the phases of the exact ground state is in general very useful to improve the efficiency of the approximated quantum Monte Carlo techniques that have to be used in presence of the sign problem. 17.18

In the triangular case, instead, the phases obtained by applying the operator (11) to a state with positive-definite amplitudes on the Ising basis, as in Eq. (10), are very far from being exact, especially in the spin-isotropic limit (see Sec. 3.2.1).

\subsubsection{Ordering of the energy levels}

It follows from the spin rotational invariance of the Hamiltonian (3) that each energy level, $E(S)$, belonging to the total spin $S$ subspace is $(2 S+1)$-fold degenerate: in any $S$ sector there is a degenerate level for each value of $M$ in the range $-S \leq$ 
$M \leq S$. Therefore, in a given $M$ subspace every energy eigenstate with total spin $S \geq|M|$ must be contained. In the hypothesis stated above, it is possible to prove that the lowest energy in each $M$ subspace belongs to $S=M$, i.e., it has the minimum total spin allowed.

In order to prove this result we will show that the ground state of $\hat{\mathcal{H}}$ in an $M$ subspace is not orthogonal to the ground state of a rotational invariant soluble Hamiltonian, which is known to belong to the $S=M$ sector. Therefore, so does the former since two eigenfunctions having different quantum numbers are in general orthogonal. Let us consider the infinite-range Heisenberg Hamiltonian on a bipartite lattice

$$
\hat{\mathcal{H}}_{\infty}=J \sum_{i \in A, j \in B} \hat{\mathbf{S}}_{i} \cdot \hat{\mathbf{S}}_{j}
$$

with $J$ positive constant. This Hamiltonian is rotationally invariant and exactly soluble since it is equivalent to a two spin problem:

$$
\hat{\mathcal{H}}_{\infty}=J\left(\hat{\mathbf{S}}^{2}-\hat{\mathbf{S}}_{A}^{2}-\hat{\mathbf{S}}_{B}^{2}\right)
$$

where $\hat{\mathbf{S}}_{A}^{2}$ and $\hat{\mathbf{S}}_{B}^{2}$ are the total spin squared on the $A$ and $B$ sublattices, respectively. The eigenvalues of this special Hamiltonian are

$$
E_{\infty}(S)=\frac{J}{2}\left[S(S+1)-S_{A}\left(S_{A}+1\right)-S_{B}\left(S_{B}+1\right)\right],
$$

and are monotonically increasing with the total spin $S$. Then, the ground state of $\hat{\mathcal{H}}_{\infty}$, in each $M$ subspace, has $S=M$ total spin. Moreover, both $\hat{\mathcal{H}}$ and $\hat{\mathcal{H}}_{\infty}$ satisfy the requirements for the Marshall-Peierls sign rule. Hence, their ground state in any $M$ sector are not orthogonal since their overlap involves the sum of positive numbers. It follows that they have necessarily the same total spin quantum number $S=M$. Therefore in a given $M$ subspace the lowest energy of the Heisenberg Hamiltonian has the minimum total spin allowed.

This implies in turn that $E(S)<E(S+1)$. In fact, among the degenerate eigenfunctions with $E(S+1)$, there is a representative in the $M=S$ subspace. The latter has not the minimum total spin allowed for that subspace and therefore it has an energy higher than $E(S)$. This proves that the energy levels of the Heisenberg antiferromagnet (3) increase monotonically with the total spin and, in particular, that the absolute ground state is a singlet and non-degenerate (Lieb-Mattis property). ${ }^{b}$

The above proof on the ordering of the energy levels is a direct consequence of the Marshall-Peierls sign rule and therefore it breaks in presence of frustration. However the Lieb-Mattis property turns out to be verified even for frustrated spin systems. In particular, for symmetry reasons, the ground state on any finite size of the spin-isotropic Heisenberg antiferromagnet is believed to possess all the symmetries of the Hamiltonian and in particular to be a singlet, rotationally invariant, and

\footnotetext{
${ }^{b}$ In fact, having $S=0$, it has only a representative in the $M=0$ subspace
} 
non-degenerate. 25 Even if there is no rigorous theorem proving this property in general, the latter turns out to be true on a finite size whenever the cluster is large enough, it has an even number of sites, and the boundary conditions do no frustrate the antiferromagnetic long-range order. 13 In any case, however, these symmetry properties concern in general the ground states on finite sizes only. In the thermodynamic limit, the situation can change drastically if there is no gap in the excitation spectrum. In this case, in fact, a family of excited states collapses onto the ground state and may break its symmetric character. This will be illustrated in the following sections.

\subsection{Order parameters and susceptibilities}

A zero-temperature spontaneously broken symmetry occurs when the ground state has a lower degree of symmetry than the corresponding Hamiltonian. In this case, one can define an extensive operator, $\hat{O}$, breaking some symmetry of the Hamiltonian and such that the so-called order parameter, i.e., the groundstate expectation value $m=\left\langle\psi_{0}|\hat{O}| \psi_{0}\right\rangle / N$, has a finite value. In general, whenever the symmetry-breaking operator $\hat{O}$ does not commute with the Hamiltonian, the symmetry breaking can happen only in the thermodynamic limit. In fact, in that case, the ground-state expectation value of $\hat{O}$ is zero on any finite size by symmetry. This will be the case for the symmetry-breaking operators considered in this paper.

The occurrence of a spontaneously broken symmetry can be detected by adding to the Hamiltonian $\hat{\mathcal{H}}$ an ordering field $\delta$ :

$$
\hat{\mathcal{H}}_{\delta}=\hat{\mathcal{H}}-\delta \hat{O}
$$

Since on a finite size the ground-state expectation value of $\hat{O}$ vanishes for $\delta=0$, the ground-state energy per site has corrections proportional to $\delta^{2}$,

$$
e(\delta) \simeq e_{0}-\frac{1}{2} \chi_{O} \delta^{2}
$$

$\chi_{O}$ being the (positive-definite) generalized susceptibility associated to the operator $\hat{O}$, namely:

$$
\chi_{O}=\frac{2}{N}\left\langle\psi_{0}\left|\hat{O}\left(E_{0}-\hat{\mathcal{H}}\right)^{-1} \hat{O}\right| \psi_{0}\right\rangle
$$

where $E_{0}$ is the ground-state energy of $\hat{\mathcal{H}}$.

If symmetry breaking occurs in the thermodynamic limit then

$$
\lim _{\delta \rightarrow 0} \lim _{N \rightarrow \infty} \frac{1}{N}\left\langle\psi_{0}|\hat{O}| \psi_{0}\right\rangle=m \neq 0,
$$

and the finite-size susceptibility has to diverge with the system size. In fact, by the Hellmann-Feynman theorem, the ground-state expectation value of $\hat{O}$ at finite field is $\langle\hat{O}\rangle_{\delta} / N=-d e(\delta) / d \delta$, so that, if symmetry breaking occurs in the thermodynamic limit, an infinitesimal field $\delta$ must give a finite $\langle\hat{O}\rangle_{\delta} / N \sim \chi_{O} \delta$ implying that the 
susceptibility has to diverge in the thermodynamic limit. Moreover, it is possible to show that the finite-size susceptibility must diverge at least as the volume squared $N^{2}$. This will be proven in the next section.

\subsubsection{Exact bounds on the susceptibilities}

Since on a finite size $\left\langle\psi_{0}|\hat{O}| \psi_{0}\right\rangle=0$, it is convenient to introduce as the order parameter the quantity $p=\sqrt{\left\langle\psi_{0}\left|\hat{O}^{2}\right| \psi_{0}\right\rangle / N^{2}}$. The latter is finite in general on any finite size and extrapolate to a finite value in the thermodynamic limit in presence of long-range order, i.e., whenever $m$, given by Eq. (20), is finite. In this section we will show that, whenever symmetry breaking occurs in the thermodynamic limit, the corresponding susceptibility must diverge as $N \rightarrow \infty$ and, in particular, it is bounded from below by the order parameter times the system volume squared, namely $\chi_{O}>\operatorname{const} p^{4} N^{2}$.

Let us define the following decomposition:

$$
p^{2}=\frac{1}{N^{2}}\left\langle\psi_{0}\left|\hat{O}^{2}\right| \psi_{0}\right\rangle=\frac{1}{N^{2}} \sum_{n \neq 0}\left|\left\langle\psi_{0}|\hat{O}| \psi_{n}\right\rangle\right|^{2}=\frac{1}{N} \int d \omega S(\omega)
$$

with

$$
S(\omega)=\frac{1}{N} \sum_{n \neq 0}\left|\left\langle\psi_{0}|\hat{O}| \psi_{n}\right\rangle\right|^{2} \delta\left(\omega-\omega_{n}\right)
$$

where we have introduced a complete set of eigenstates of the Hamiltonian $\left|\psi_{n}\right\rangle$ with eigenvalues $E_{n}$, we have used the symmetry of the ground state (i.e., $\left\langle\psi_{0}|\hat{O}| \psi_{0}\right\rangle=0$ ) and set $\omega_{n}=E_{n}-E_{0}$. By the Cauchy-Schwartz inequality we have:

$$
\begin{aligned}
\int d \omega S(\omega) & =\int d \omega \omega^{1 / 2} S(\omega)^{1 / 2} \omega^{-1 / 2} S(\omega)^{1 / 2} \\
& \leq\left[\int d \omega \omega S(\omega) \int d \omega \omega^{-1} S(\omega)\right]^{1 / 2}
\end{aligned}
$$

Now,

$$
\int d \omega \omega^{-1} S(\omega)=\frac{1}{N} \sum_{n \neq 0} \frac{1}{\omega_{n}}\left|\left\langle\psi_{0}|\hat{O}| \psi_{n}\right\rangle\right|^{2} \equiv \frac{\chi_{O}}{2}
$$

where $\chi_{O}$ by Eq. (19) is the susceptibility associated to the operator $\hat{O}$. In addition it is straightforward to show that

$$
\int d \omega \omega S(\omega)=\frac{1}{N} \sum_{n \neq 0} \omega_{n}\left|\left\langle\psi_{0}|\hat{O}| \psi_{n}\right\rangle\right|^{2}=\frac{1}{2 N}\langle[\hat{O},[\hat{\mathcal{H}}, \hat{O}]]\rangle \equiv \frac{f_{0}}{2},
$$

so that, using Eqs. (23) and (24), we have

$$
p^{2}=\frac{1}{N} \int d \omega S(\omega) \leq \frac{1}{2 N} \sqrt{\chi_{O} f_{0}} .
$$


Hence, we have obtained the following lower bound for the susceptibility:

$$
\chi_{O} \geq \frac{4 p^{4}}{f_{0}} N^{2} .
$$

Therefore, if the order parameter is finite in the thermodynamic limit, the susceptibility must diverge at least as the volume squared, provided $f_{0}$ is a constant. This happens whenever the commutator of $\hat{\mathcal{H}}$ and $\hat{O}$ is an extensive quantity as it is the case for the magnetization in the Heisenberg model and for all the symmetrybreaking operators treated in this work.

Moreover it is also possible to construct an upper bound for the generalized susceptibility $\chi_{O}$ associated to a symmetry-breaking operator $\hat{O}$. In fact, using Eqs. (24) and (21), we have

$$
\frac{\chi_{O}}{2}=\frac{1}{N} \sum_{n \neq 0} \frac{1}{\omega_{n}}\left|\left\langle\psi_{0}|\hat{O}| \psi_{n}\right\rangle\right|^{2} \leq \frac{1}{\Delta} \int d \omega S(\omega)=\frac{N p^{2}}{\Delta}
$$

where $\Delta$ is the energy gap between the ground state and the first excitation. An energy gap in the thermodynamic excitation spectrum is therefore incompatible with a spontaneously broken symmetry. The physical meaning of this quite general result is the following: in presence of a gap in the excitation spectrum, the ground state, which has generally all the symmetries of the Hamiltonian on any finite size, has clearly no mean to develop a spontaneously broken symmetry in the thermodynamic limit. In this case, the susceptibility is bounded [Eq. (28)]. In contrast, in presence of a gapless excitation spectrum, a family (or a tower) of excited states can collapse in the thermodynamic limit onto the ground state and can break its symmetric character. In fact, these states acquire in general a phase factor under some operation of the symmetry group of the Hamiltonian and they can give rise to a symmetry-broken superposition. Whenever this happens, the related susceptibility must diverge as the volume squared [Eq. (27)]. In this case from Eqs. (27) and (28) we get a remarkable relation for the size-dependence of the spin gap, namely

$$
\Delta \leq \frac{f_{0}}{2 p^{2}} N^{-1} .
$$

The mechanism underlying the spontaneous symmetry breaking leading to the onset of long-range Néel order in the thermodynamic ground state of a quantum antiferromagnet will be discussed in more detail in the following section.

\subsection{Néel order and Anderson's towers of states}

As we have already noticed, the occurrence of a spontaneous symmetry breaking in the thermodynamic ground state, can be evidenced from the structure of the finite-size energy spectrum.

On any finite size, the ground state of a quantum antiferromagnet is generally believed to be a singlet, rotationally invariant and non-degenerate, i.e., non-magnetic. 
This is rigorously stated by the Lieb-Mattis theorem only for the Heisenberg square antiferromagnet but nonetheless it can be numerically verified on small clusters also in presence of frustration for the triangular Heisenberg antiferromagnet 13 and for the $J_{1}-J_{2}$ model itself.26 Therefore, as originally pointed out by Anderson, 11 the spontaneously symmetry breaking mechanism necessarily involves the low-energy portion of the excitation spectrum: in particular, a whole tower of states has to collapse in the thermodynamic limit onto the ground state faster than the low-lying excitations involving a spatial modulation of the classical Néel state (the so-called magnons). In fact, since in general these states acquire phase factors under rotations in the spin space, they can sum up to a nontrivial state in which the spins point in a definite direction, giving rise to Néel-like long-range order.

In particular, it is well known27-30 that in this case the low-lying excited states of energy $E(S)$ and spin $S$ are predicted to behave as the spectrum of a free quantum rotator (or quantum top) as long as $S \ll \sqrt{N}$,

$$
E(S)-E_{0}=\frac{S(S+1)}{2 I N},
$$

where $E_{0}=E(0)$ is the energy of the ground state, $\left|\psi_{0}\right\rangle$, and $I$ is known as the momentum of inertia per site and is an intensive quantity.

This equation, which is in agreement with the bound (29), can be justified in a semiclassical picture of the long-range ordered ground state of a quantum antiferromagnet.13 To this purpose, let us consider the nearest-neighbor Heisenberg antiferromagnet on the square lattice, and separate in the Fourier transformed Hamiltonian the $\mathbf{k}=0$, and $\mathbf{Q}=(\pi, \pi)$ contributions (i.e., the only ones allowed by the sublattice translation invariance of the classical Néel state) from the others:

$$
\hat{\mathcal{H}}=\hat{\mathcal{H}}_{0}+\hat{\mathcal{V}},
$$

where

$$
\hat{\mathcal{H}}_{0}=\frac{4 J}{N}\left(\hat{\mathbf{S}}^{2}-\hat{\mathbf{S}}_{A}^{2}-\hat{\mathbf{S}}_{B}^{2}\right),
$$

$\hat{\mathbf{S}}^{2}$ is the total spin square, and $\hat{\mathbf{S}}_{A}^{2}$ and $\hat{\mathbf{S}}_{B}^{2}$ are the total spin square of the A and B sublattices, respectively;

$$
\hat{\mathcal{V}}=2 J \sum_{\mathbf{k} \neq 0, \mathbf{Q}} \gamma_{\mathbf{k}} \hat{\mathbf{S}}_{\mathbf{k}} \cdot \hat{\mathbf{S}}_{-\mathbf{k}}
$$

where $\hat{\mathbf{S}}_{\mathbf{k}}=1 / \sqrt{N} \sum_{i} \hat{\mathbf{S}}_{i} \exp \left(\mathbf{k} \cdot \mathbf{r}_{i}\right), \mathbf{r}_{i}$ is the position of the site $i$, and $\gamma_{\mathbf{k}}=$ $\left(\cos k_{x}+\cos k_{y}\right) / 2$. For each value of the total spin $S$ the lowest eigenstate of $\hat{\mathcal{H}}_{0}$ is the classical-like state fully polarized on each magnetic sublattice with energy:

$$
E_{0}(S)=-\frac{1}{4}(N+4)+\frac{4 J S(S+1)}{N} .
$$

This is in agreement with the quantum top law (30). Of course, since $\hat{\mathbf{S}}_{A}^{2}$ and $\hat{\mathbf{S}}_{B}^{2}$ do not commute with $\hat{\mathcal{V}}$, such eigenstates are not eigenstates of $\hat{\mathcal{H}}$. Nonetheless we 
can look at them as the first approximation to the low-lying excited states in each $S$ sector. The perturbation $\hat{\mathcal{V}}$ dresses these classical-like states with quantum fluctuations decreasing the average value of the sublattice magnetization and lowering their energy towards the exact result. However, as long as the energy scale of these states is well separated by the low-lying excitations with $\mathbf{k} \neq 0, \mathbf{Q}$ such renormalizations are not expected to modify the behavior $(30)$. These excitations are known as magnons or spin-waves (see Sec. 3.1), and involve a spatial modulation of the classical Néel state. In the Heisenberg antiferromagnet the dispersion relation of the softest magnons is linear in the wavevector so that, in two dimensions, they have an energy scaling as $1 / \sqrt{N}$. This implies that the constraint on the value of $S$ for the validity of Eq. (30) is $S \ll \sqrt{N}$.

The quantum top law is similar to the definition of the uniform spin susceptibility. 31 The latter, in fact, can be calculated, by taking first the infinite-volume limit of the energy per site $e(m)=E(S) / N$ at fixed magnetization $m=S / N$ and then letting $m \rightarrow 0$ in the expansion

$$
e(m)=e_{0}+\frac{m^{2}}{2 \chi}
$$

which is quite similar to Eq. (30). However an identification between $I$ and $\chi$ is possible if the excitation spectrum smoothly connects the low-energy portion which corresponds to total spin $S \tilde{\mathcal{O}}(1)$, with the regime of macroscopic spin excitations: $S \sim m N$ (with $m \ll 1$ ). 32 This is an highly nontrivial statement which is actually verified by the underlying low-energy effective model of the quantum antiferromagnet, known as nonlinear $\sigma$ model $(\mathrm{NL} \sigma \mathrm{M}) .25 .29 .30 .3233$ Therefore, the quantity

$$
\frac{1}{\chi_{S}}=2 N \frac{E(S)-E_{0}}{S(S+1)}
$$

must approach the inverse of the spin susceptibility for infinite size and for any spin excitation with $S \ll N$.

\subsection{Resonating Valence Bond states}

A simple and clear picture of a non-magnetic ground state can be given in terms of the so-called Resonating Valence Bond (RVB) wavefunctions.34 Here, for simplicity, we will restrict ourselves to the case of the spin-half square antiferromagnet even if these states can be used also for a generic value of the spin $s$ (Ref. 35 ) and different lattice geometries. 30

The RVB wavefunctions are linear superpositions of valence bond states in which each spin forms a singlet bond with another spin on the opposite sublattice. These states form in general a (overcomplete) basis of the $S=0$ subspace so that any singlet wavefunction can be represented in terms of them. In particular, with such RVB wavefunctions it is possible to describe either a long-range ordered or a nonmagnetic state by varying the bond-length distribution. 35 In order to clarify this 


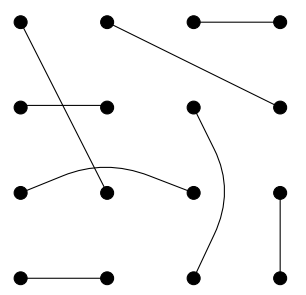

(a)

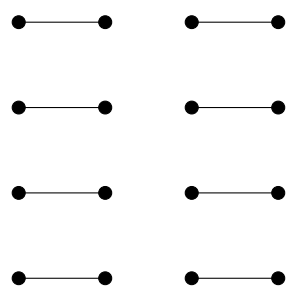

(b)

Figure 3: An example of a spin liquid (a) and of a symmetry-broken (b) nonmagnetic RVB state. Each stick represents a singlet bond.

point, let us consider the following class of RVB wavefunctions for a system of $N$ spins:

$$
\left|\psi_{R V B}\right\rangle=\sum_{i_{\alpha} \in A, j_{\beta} \in B} h\left(r_{1}\right) \ldots h\left(r_{n}\right)\left(i_{1} j_{1}\right) \ldots\left(i_{n}, j_{n}\right)
$$

where $n=N / 2, r_{m}$ is the distance between the spins forming the $m^{t h}$ singlet bond $\left(i_{m} j_{m}\right)$, and $h\left(r_{m}\right)$ is a bond weight factor, function of its length. The latter wavefunction has no long-range order whenever the short-ranged bonds are the dominant one in the superposition (37). More precisely, it has been numerically shown by Liang, Doucot and Anderson 37 that the RVB state (37) has no longrange antiferromagnetic order for bonds that decay as rapidly as $h(r) \sim r^{-p}$, with $p \geq 5$. Instead, if the weight functions decay slowly enough with the length of the bond, then the RVB wavefunction has a finite value of the thermodynamic order parameter squared. In particular, if the weight factors $h(r)$ are independent of the bond length, the RVB wavefunction is the projection of the Néel state onto the singlet subspace.

Therefore, the simplest physical picture of a non-magnetic ground state can be given in terms of a RVB wavefunction with short-ranged bonds. In addition, such bonds can be either homogeneously spatially distributed on the lattice, with short-range correlations among each other (spin liquid) [Fig. 3 (a)], or they can break some symmetries of the Hamiltonian, with the dimers frozen in some special patterns [Fig. 3 (b)]. In Sec. 4.3 we will provide a possible example of the latter situation.

\section{The Triangular Heisenberg Antiferromagnet}

Historically the antiferromagnetic spin-1/2 Heisenberg model on the triangular lattice was the first proposed Hamiltonian for a microscopic realization of a nonmagnetic ground state. 34.38 This is due to the fact that in this system the usual antiferromagnetic alignment between spins is hindered by the geometry of the lattice 
so that the minimum energy configuration, the $2 \pi / 3$ Néel state (Fig. 1), has an energy twice larger than that on the square lattice and therefore is far less stable.

However, despite a strong theoretical effort, the question of whether the combined effect of frustration and quantum fluctuations in the ground state of the triangular Heisenberg antiferromagnet favors a disordered RVB state or long-range Néel type order has been under debate for many years. 13 .25. $36.39-54$

Spin-wave calculation $\$ 51.52$ predict an important reduction (by about one-half) of the sublattice magnetization by quantum fluctuations. In addition, perturbation theory, 50 series expansions, 46 and high-temperature calculations 47 suggest that the spin-wave calculations possibly underestimate the renormalization of the order parameter, but do not come to a definite conclusion about the nature of ground state, From the numerical point of view, exact diagonalization (ED) results, $13.39 .40-43$ which are limited to small lattice sizes, have been interpreted both against 43 and in favor 13 of the presence of long-range Néel order in the thermodynamic ground state. In any case, this approach, combined with the careful analysis of the symmetry properties of the low-energy excited states proposed by Bernu and co-workers, 13 have provided very important evidence pointing towards a magnetic ground state: the spectra of the lowest energy levels order with increasing total spin, a reminiscence of the Lieb-Mattis theorem (see Sec. 2.1) for bipartite lattices, and are consistent with the symmetry of the classical order parameter. However, these very clear ED results cannot rule out that for large sizes quantum fluctuations could drive the system into a non-magnetic phase and therefore cannot be considered as conclusive. In addition, standard stochastic numerical methods, which usually allow one to handle large samples, clash with the sign problem numerical instability so that a definite answer on the ground-state properties of the triangular Heisenberg antiferromagnet has been lacking for many years.

In this section we will tackle the problem of the existence of long-range Néel order in the ground state of the triangular Heisenberg antiferromagnet using the finitesize spin-wave theory, 10 ED, and Green function Monte Carlo (GFMC) methods. In the first part we apply the finite-size spin-wave theory to the triangular Heisenberg antiferromagnet, we then show how to construct within this framework the lowlying excited states, and finally derive a simple spin-wave variational wavefunction. The good agreement between the ED results and the finite-size spin-wave theory will support the reliability of the spin-wave expansion in describing not only the groundstate properties but also the low-energy spin excitations of the Heisenberg model even in presence of frustration. The second part will deal with the quantum Monte Carlo results. Data for the spin gap and for the antiferromagnetic order parameter will be presented for fairly large system sizes (up to 144 sites), providing a robust evidence for a gapless excitation spectrum and for the existence of long-range Néel order in the thermodynamic ground state of the model.

\subsection{Finite-size spin-wave theory}

Several attempts to generalize spin-wave theory to finite sizes can be found in the 
literature 10,55,56 Here we will follow the method proposed by Zhong and Sorella in Ref. 10 which allows one to deal with finite clusters avoiding the spurious Goldstone modes divergences in a straightforward way, and, in particular, without imposing any ad hoc holonomic constraint on the sublattice magnetization.55 56

\subsubsection{Application to the triangular antiferromagnet}

Assuming the classical $\mathbf{Q}=(4 \pi / 3,0)$ magnetic structure lying in the $x y$ plane, the first step of the derivation is to apply the unitary transformation given by Eq. (11), which defines a spatially varying coordinate system $\left(x^{\prime} y^{\prime} z^{\prime}\right)$ in such a way that the $x^{\prime}$-axis points on each site along the local Néel direction. The transformed Heisenberg Hamiltonian reads:

$$
\begin{aligned}
\hat{\mathcal{U}}^{\dagger} \hat{\mathcal{H}} \hat{\mathcal{U}} & =J \sum_{\langle i, j\rangle}\left[\cos \left(\mathbf{Q} \cdot \mathbf{r}_{i, j}\right)\left(\hat{S}_{i}^{x^{\prime}} \hat{S}_{j}^{x^{\prime}}+\hat{S}_{i}^{y^{\prime}} \hat{S}_{j}^{y^{\prime}}\right)\right. \\
& \left.+\sin \left(\mathbf{Q} \cdot \mathbf{r}_{i, j}\right)\left(\hat{S}_{i}^{x^{\prime}} \hat{S}_{j}^{y^{\prime}}-\hat{S}_{i}^{y^{\prime}} \hat{S}_{j}^{x^{\prime}}\right)+\hat{S}_{i}^{z^{\prime}} \hat{S}_{j}^{z^{\prime}}\right]
\end{aligned}
$$

where $J$ is the (positive) exchange constant between nearest neighbors, the indices $i, j$ label the points $\mathbf{r}_{i}$ and $\mathbf{r}_{j}$ on the $N$-site triangular lattice, $\mathbf{r}_{i, j}=\mathbf{r}_{i}-\mathbf{r}_{j}$, and the quantum spin operators satisfy $\left|\hat{\mathbf{S}}_{i}\right|^{2}=s(s+1)$. In the new reference frame the spins in the classical configuration are ferromagnetically aligned so that, using Holstein-Primakoff transformation for spin operators to order $1 / s$,

$$
\hat{S}_{i}^{x^{\prime}}=s-\hat{a}_{i}^{\dagger} \hat{a}_{i} \quad \hat{S}_{i}^{y^{\prime}}=\sqrt{\frac{s}{2}}\left(\hat{a}_{i}^{\dagger}+\hat{a}_{i}\right) \quad \hat{S}_{i}^{z^{\prime}}=i \sqrt{\frac{s}{2}}\left(\hat{a}_{i}^{\dagger}-\hat{a}_{i}\right),
$$

being $\hat{a}$ and $\hat{a}^{\dagger}$ the canonical creation and destruction Bose operators, after some algebra the Fourier transformed Hamiltonian results:

$$
\hat{\mathcal{H}}_{\mathrm{SW}}=E_{c l}+3 J s \sum_{\mathbf{k}}\left[A_{\mathbf{k}} \hat{a}_{\mathbf{k}}^{\dagger} \hat{a}_{\mathbf{k}}+\frac{1}{2} B_{\mathbf{k}}\left(\hat{a}_{\mathbf{k}}^{\dagger} \hat{a}_{-\mathbf{k}}^{\dagger}+\hat{a}_{\mathbf{k}} \hat{a}_{-\mathbf{k}}\right)\right]
$$

where $E_{c l}=-3 J s^{2} N / 2$ is the classical ground-state energy,

$$
A_{\mathbf{k}}=1+\gamma_{\mathbf{k}} / 2, B_{\mathbf{k}}=-3 \gamma_{\mathbf{k}} / 2 \text {, }
$$

$\gamma_{\mathbf{k}}=2\left[\cos \left(k_{x}\right)+2 \cos \left(k_{x} / 2\right) \cos \left(\sqrt{3} k_{y} / 2\right)\right] / z, \mathbf{k}$ is a vector varying in the first Brillouin zone of the lattice, and $z=6$ is the coordination number. The Hamiltonian $\hat{\mathcal{H}}_{\mathrm{SW}}$, can be diagonalized for $\mathbf{k} \neq 0, \pm \mathbf{Q}$ introducing the well-known Bogoliubov transformation, $\hat{a}_{\mathbf{k}}=u_{\mathbf{k}} \hat{\alpha}_{\mathbf{k}}+v_{\mathbf{k}} \hat{\alpha}_{-\mathbf{k}}^{\dagger}$, with

$$
u_{\mathbf{k}}=\left(\frac{A_{\mathbf{k}}+\epsilon_{\mathbf{k}}}{2 \epsilon_{\mathbf{k}}}\right)^{1 / 2}, v_{\mathbf{k}}=-\operatorname{sgn}\left(B_{\mathbf{k}}\right)\left(\frac{A_{\mathbf{k}}-\epsilon_{\mathbf{k}}}{2 \epsilon_{\mathbf{k}}}\right)^{1 / 2},
$$

where $\epsilon_{\mathbf{k}}=\sqrt{A_{\mathbf{k}}^{2}-B_{\mathbf{k}}^{2}}$ is the spin-wave dispersion relation. This diagonalization leads to:

$$
\mathcal{H}_{\mathrm{SW}}^{0}=E_{c l}+\frac{3 J s}{2} \sum_{\mathbf{k} \neq 0, \pm \mathbf{Q}}\left(\epsilon_{\mathbf{k}}-A_{\mathbf{k}}\right)+\frac{3 J s}{2} \sum_{\mathbf{k} \neq 0, \pm \mathbf{Q}} \epsilon_{\mathbf{k}}\left(\hat{\alpha}_{\mathbf{k}}^{\dagger} \hat{\alpha}_{\mathbf{k}}+\hat{\alpha}_{-\mathbf{k}}^{\dagger} \hat{\alpha}_{-\mathbf{k}}\right) .
$$


The Goldstone modes at $\mathbf{k}=\mathbf{0}$ and $\mathbf{k}= \pm \mathbf{Q}$ instead are singular, and cannot be diagonalized with a Bogoliubov transformation. For infinite systems such modes do not contribute to the integrals in Eq. (43), but in the finite-size case they are important and they must be treated separately. By defining the following Hermitian operators

$$
\begin{aligned}
\hat{Q}_{x} & =\frac{i}{2}\left(\hat{a}_{\mathbf{Q}}^{\dagger}+\hat{a}_{-\mathbf{Q}}-\hat{a}_{\mathbf{Q}}-\hat{a}_{-\mathbf{Q}}^{\dagger}\right), \\
\hat{Q}_{y} & =\frac{1}{2}\left(\hat{a}_{\mathbf{Q}}^{\dagger}+\hat{a}_{-\mathbf{Q}}+\hat{a}_{\mathbf{Q}}+a_{-\mathbf{Q}}^{\dagger}\right), \\
\hat{Q}_{z} & =i\left(\hat{a}_{0}^{\dagger}-\hat{a}_{0}\right),
\end{aligned}
$$

such that, $\left[\hat{Q}_{\alpha}, \hat{Q}_{\beta}\right]=0$ and $\left[\hat{Q}_{\alpha}, \hat{\mathcal{H}}_{\mathrm{SW}}\right]=0$ for $\alpha, \beta=x, y, z$, the contribution of the singular modes, $\hat{\mathcal{H}}_{S M}$, in Eq. (40) can be expressed in the form

$$
\hat{\mathcal{H}}_{S M}=-3 J s A_{\mathbf{0}}+3 J s \frac{A_{\mathbf{0}}}{2}\left[\hat{Q}_{x}^{2}+\hat{Q}_{y}^{2}+\hat{Q}_{z}^{2}\right] .
$$

Then, taking into account the fact that to the leading order in $1 / s, \hat{Q}_{\alpha}=\hat{S}^{\alpha} \sqrt{2 / N s}$, where $\hat{S}^{\alpha}$ are the components of the total spin, $\hat{\mathcal{H}}_{S M}$ may be also rewritten in the more physical form

$$
\hat{\mathcal{H}}_{S M}=-3 J s A_{\mathbf{0}}+3 J \frac{A_{\mathbf{0}}}{N}\left[\left(\hat{S}^{x}\right)^{2}+\left(\hat{S}^{y}\right)^{2}+\left(\hat{S}^{z}\right)^{2}\right],
$$

which clearly favors a singlet $\left(\mathbf{S}^{2}=0\right)$ ground state (for an even number of sites) being $A_{\mathbf{0}}$ positive definite. This result is a reminiscence of the Lieb-Mattis property (see Sec. 2.1) which has not been demonstrated for non-bipartite lattices. Actually, a similar result is obtained by solving exactly the three Fourier components $\mathbf{k}=\mathbf{0}, \pm \mathbf{Q}$ of the Heisenberg model 13 however, our treatment allows us to construct a formal expression for the spin-wave ground state on finite triangular lattices which keeps the correct singlet behavior. In fact, starting from the usual spin-wave ground state, composed by the $2 \pi / 3$ classical Néel order plus the zero point quantum fluctuations (i.e, zero Bogoliubov quasiparticles),

$$
|0\rangle=\prod_{\mathbf{k} \neq \mathbf{0}, \pm \mathbf{Q}} u_{\mathbf{k}}^{-1} \exp \left[\frac{1}{2} \frac{v_{\mathbf{k}}}{u_{\mathbf{k}}} \hat{a}_{\mathbf{k}}^{\dagger} \hat{a}_{-\mathbf{k}}^{\dagger}\right]|F\rangle
$$

with $|F\rangle=\prod_{i}\left|S_{i}^{x^{\prime}}=s\right\rangle$, the corresponding singlet wavefunction is obtained by projecting $|0\rangle$ onto the subspace $S=0$ :

$$
\left|\psi_{\mathrm{SW}}\right\rangle=\int_{-\infty}^{\infty} d \alpha \int_{-\infty}^{\infty} d \beta \int_{-\infty}^{\infty} d \gamma e^{i \alpha Q_{x}+i \beta Q_{y}+i \gamma Q_{z}}|0\rangle
$$

and reads $\left|\psi_{\mathrm{SW}}\right\rangle \sim e^{\left(-\hat{a}_{\mathrm{Q}}^{\dagger} \hat{a}_{-\mathbf{Q}}^{\dagger}+\frac{1}{2} \hat{a}_{0}^{\dagger} \hat{a}_{0}^{\dagger}\right)}|0\rangle$. In particular the singular modes have no contribution to the ground-state energy which reads

$$
E_{\mathrm{SW}}=E_{c l}+\frac{3 J s}{2} \sum_{\mathbf{k}}\left(\epsilon_{\mathbf{k}}-1\right)
$$


while the computation of the order parameter requires their remotion:

$$
\hat{m}^{\dagger}=\sqrt{\left\langle\left(\hat{S}_{i}^{x^{\prime}}\right)^{2}\right\rangle}=s-\frac{1}{N} \sum_{\mathbf{k} \neq \mathbf{0}, \pm \mathbf{Q}} v_{\mathbf{k}}^{2} .
$$

For $s=1 / 2$, the previous spin-wave calculation predicts a very good quantitative agreement with exact results on small clusters $(N \leq 36)$ of both ground-state energy and sublattice magnetization.57 The agreement is even more remarkable as far as the low-lying excited states are concerned, as it will be shown in the following section.

\subsubsection{Low-energy spin-wave spectrum}

In this section, we show how to construct the low-lying energy spectra $E(S)$ for finite systems where $S$ represents the total spin. So far, we have performed a standard spin-wave expansion whose relevant quantum number is $s$. Thus, computing $E(S)$ is not straightforward and require a little more involved calculation. Following Lavalle, Sorella and Parola 32 a magnetic field in the $z$-direction is added to stabilize the desired total spin excitation $S$ :

$$
\hat{\mathcal{H}}_{\mathrm{SW}}^{h}=\hat{\mathcal{H}}_{\mathrm{SW}}-h s \sum_{i} \hat{S}_{i}^{z} .
$$

Classically, for magnetic fields not large enough to induce a spin-flop transition, the new solution is the $2 \pi / 3$ Néel order canted by an angle $\theta$ along the direction of the field $h$. In order to develop a spin-wave calculation, a new rotation around $y^{\prime}$-axis

is performed on the spin operators and it can be proven that $\mathcal{H}_{\mathrm{SW}}^{h}$ takes the same form of Eq. (40) with renormalized coefficients $A_{\mathbf{k}}$ and $B_{\mathbf{k}}$ :

$$
A_{\mathbf{k}}^{h}=1+\gamma_{\mathbf{k}}\left[\frac{1}{2}-\frac{3}{2}\left(\frac{2 h}{z 3 J}\right)^{2}\right], B_{\mathbf{k}}^{h}=-\frac{3}{2} \gamma_{\mathbf{k}}\left[1-\left(\frac{2 h}{z 3 J}\right)^{2}\right],
$$

being $2 h / z 3 J=\sin \theta$. For $h \neq 0$ the only singular mode is $\mathbf{k}=0$ (associated to the rotation invariance in the $x y$ plane) and its contribution is given by

$$
\mathcal{H}_{S M}=-\frac{3 J s}{2} A_{\mathbf{0}}^{h}+3 J \frac{A_{\mathbf{0}}^{h}}{N}\left(S^{z}-N s \sin \theta\right)^{2},
$$

which now favors a value of $S^{z}$ consistent with the applied field, at the classical level.

The Hellmann-Feynman theorem relates the total spin $S=N\left\langle S_{i}^{z}\right\rangle$ of the excitation to the magnetic field $h$ as it follows:

$$
\left\langle S_{i}^{z}\right\rangle=-\frac{1}{N s} \frac{\partial}{\partial h} E(h)=s \frac{2 h}{z 3 J}\left[1+\frac{1}{2 N s} \sum_{\mathbf{k} \neq 0} \gamma_{\mathbf{k}} \sqrt{\frac{A_{\mathbf{k}}^{h}+B_{\mathbf{k}}^{h}}{A_{\mathbf{k}}^{h}-B_{\mathbf{k}}^{h}}}\right]
$$

where

$$
E(h)=E_{c l}-\frac{1}{2}(s h)^{2} \frac{2 N}{3 z J}-3 J s \frac{N}{2}+\frac{3 J s}{2} \sum_{\mathbf{k}} \epsilon_{\mathbf{k}}^{h} .
$$



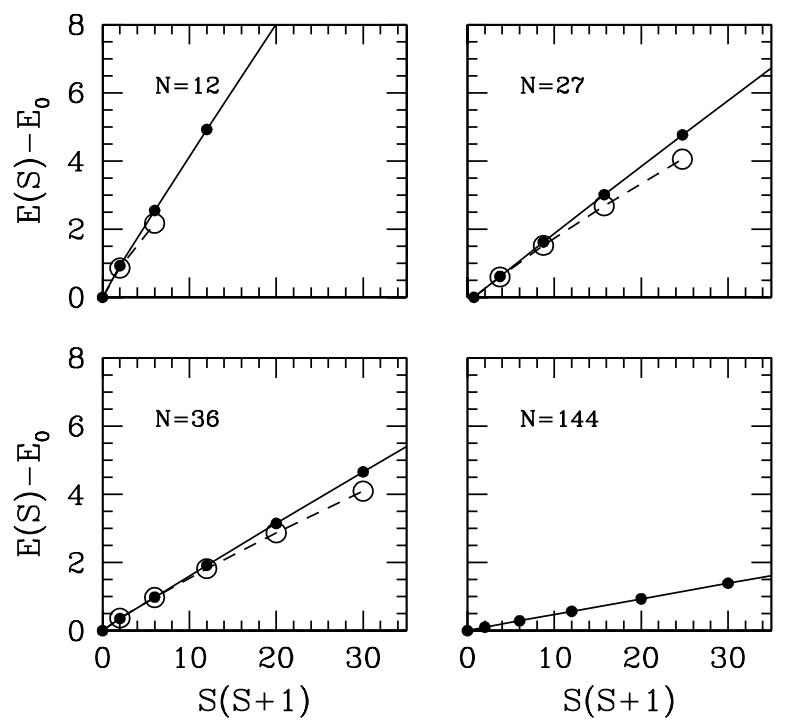

Figure 4: Spin-wave (full dots and continuous line) and exact (empty dots and dashed lines) low-energy spectra as a function of $\left|\mathbf{S}^{2}\right|=S(S+1)$ for $N=$ $12,27,36,144$ and $s=1 / 2$.

is the spin-wave energy in presence of the field and $\epsilon_{\mathbf{k}}^{h}=\sqrt{\left(A_{\mathbf{k}}^{h}\right)^{2}-\left(B_{\mathbf{k}}^{h}\right)^{2}}$. In particular, the first term in $(s h)^{2}$ in Eq. (55) gives the classical uniform spin susceptibility $\chi_{c l}=1 / 9 \mathrm{~J}$, while taking the whole expression the known spin-wave result $53 \chi_{\mathrm{S}} \mathrm{L} / \chi_{c l}=1-0.449 / 2 s$ is recovered. Finally, as suggested by Lavalle and co-workers, 32 given the value $S$, the corresponding values of $h$ and of $E(h)$ can be found with Eqs. (54) and (55), and the energy of the spin excitation $E(S)$ can be calculated, at fixed $s$, with a Legendre transformation $E(S)=E(h)+h s S 31$

As explained in Sec. 2.3, the occurrence of a symmetry breaking in the ground state for $N \rightarrow \infty$ can be evidenced from the structure of the finite-size energy spectra. In particular, when long-range order is present in the thermodynamic limit, the low-lying excited states of energy $E(S)$ and spin $S$ are predicted to behave as the spectrum of a free quantum rotator (30) as long as $S \ll \sqrt{N}$. Actually, on the triangular lattice the quantum-top effective Hamiltonian displays a correction due to the anisotropy of the susceptibility tensor 13 However, in the following we will consider only the leading contribution (30) which depends on the perpendicular susceptibility. Fig. 1 shows $E(S)$ vs $S(S+1)$ calculated within the spin-waye, theory compared with the exact diagonalization results of Bernu and co-workers 13 Remarkably the spin-wave theory turns out to be accurate in reproducing the low-energy spectrum in the whole range of sizes. Furthermore, we can extend our calculation to the thermodynamic limit and observe easily the collapse of a macroscopic number of states with different $S$ to the ground state as $N \rightarrow \infty$. This clearly gives rise to a 

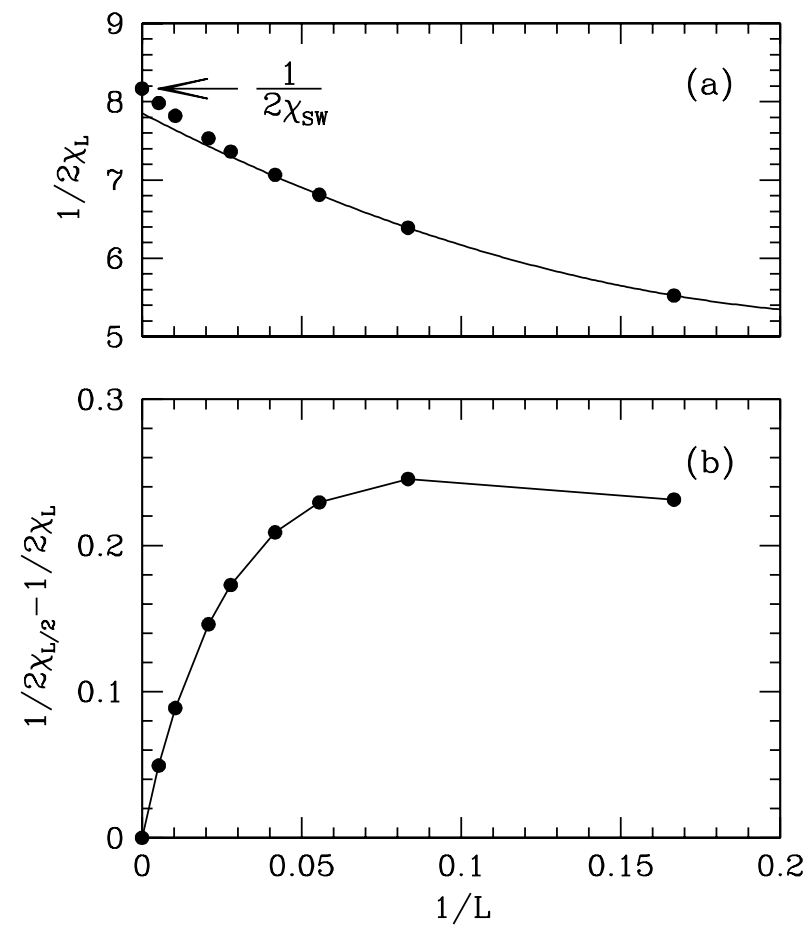

Figure 5: Size dependence of $1 / 2 \chi_{L}$ (a) and of $1 / 2 \chi_{L / 2}-1 / 2 \chi_{L}$ (b) obtained according to Eq. (56) using the $(s=1 / 2)$ spin-wave excitation spectra. The continuous line is a quadratic fit for $L<18$ in (a) and a guide for the eye in (b).

broken $\mathrm{SU}(2)$ symmetry ground state, as expected within the spin-wave framework.

In addition, as explained in Sec. 2.3, whenever the quantum top law (30) is verified, the quantity

$$
\left[2 \chi_{S}\right]^{-1}=N E(S)[S(S+1)]^{-1},
$$

should approach the physical inverse susceptibility $1 / 2 \chi_{\mathrm{SW}}$ for infinite size and for any spin excitation $S \ll N$. This feature is clearly present in the spin-wave theory and it is shown in Fig. 5 (a) where the $1 / 2 \chi_{S}$ is plotted for $S=L \equiv \sqrt{N}$ and approaches the predicted value $\left(1 / 2 \chi_{\mathrm{SW}}=8.167\right)$, even if the correct asymptotic scaling $1 / 2 \chi_{L} \simeq 1 / 2 \chi_{\mathrm{SW}}+a / L+b / L^{2}$ turns out to be satisfied only for very large sizes $(L \geq 36)$. Such feature is also shared by the Heisenberg antiferromagnet on the square lattice where a similar spin-wave analysis has allowed the authors of Ref. 32 to account for the anomalous finite-size spectrum resulting from an accurate quantum Monte Carlo calculation. Furthermore, similarly to the latter case, a nonmonotonic behavior of $1 / 2 \chi_{L / 2}-1 / 2 \chi_{L}$ [Fig. 司(b)], which should extrapolate to 0 as $1 / L$ according to the quantum top law, persists also in presence of the frustration within the spin-wave approximation and is likely to be a genuine feature of the 
Heisenberg model.

\subsubsection{Spin-wave variational wavefunctions}

In a GFMC calculation it is in general important to start from an accurate variational guess of the ground-state wavefunction. So far, many wavefunctions have been proposed in the literature $36.44,48$ and the lowest ground-state energy estimation was obtained with the long-range ordered type.36. 45

The simplest starting point for constructing a long-range ordered wavefunction is of course the classical Néel state. Since on finite-size the ground state is expected to be rotationally invariant, the Néel state should be projected onto the $\mathbf{S}^{2}=0$ subspace. However it is in general numerically very difficult to perform the projection onto a total spin subspace so that only the projection onto the subspace with $S^{z}=0$ (a quantum number of the states of the chosen basis) is usually performed. Quantum correction to this classical wavefunction can be introduced by means of a Jastrow factor containing all the two-spin correlations

$$
\left|\psi_{G}\right\rangle=\hat{P}_{0} \exp \left(\frac{\eta}{2} \sum_{i, j} v(i-j) \hat{S}_{i}^{z} \hat{S}_{j}^{z}\right)|N\rangle
$$

where $\hat{P}_{0}$ is the projector onto the $S^{z}=0$ subspace. Starting from the spin-wave ground state (48) it is possible to derive 58 a simple variational wavefunction which is both accurate and easily computable also when used for importance sampling in a quantum Monte Carlo calculation 18 Such wavefunction is defined for any $s$ in the correct Hilbert space of the spin operators and reduces for $s \rightarrow \infty$ to the spin wave form (48).

To this purpose let us consider the following variational wavefunction

$$
\left|\tilde{\psi}_{G}\right\rangle=\hat{P}_{0} \exp \left[\frac{1}{s} \sum_{\mathbf{k} \neq 0} g_{\mathbf{k}} \hat{S}_{\mathbf{k}}^{z} \hat{S}_{-\mathbf{k}}^{z}\right]|F\rangle,
$$

where $\hat{S}_{\mathbf{k}}^{z}=N^{-1 / 2} \sum_{j} e^{-i \mathbf{k} \cdot \mathbf{r}_{j}} \hat{S}_{j}^{z}$. The spin-wave $s \rightarrow \infty$ limit of the wavefunction (58) can be easily carried out by means of a Hubbard-Stratonovich transformation 6 and leads to (neglecting an unessential normalization)

$$
\left|\tilde{\psi}_{G}\right\rangle=\hat{P}_{0} \exp \left[-\frac{1}{2} \sum_{\mathbf{k} \neq 0} \frac{g_{\mathbf{k}}}{1-g_{\mathbf{k}}} \hat{a}_{\mathbf{k}}^{\dagger} \hat{a}_{-\mathbf{k}}^{\dagger}\right]|F\rangle .
$$

By requiring that $\left|\psi_{G}\right\rangle$ reduces to the spin-wave wavefunction (48) for $s \rightarrow \infty$ one obtains for $g_{\mathbf{k}}$

$$
g_{\mathbf{k}}=\frac{v_{\mathbf{k}}}{v_{\mathbf{k}}-u_{\mathbf{k}}}=1-\sqrt{\frac{1+2 \gamma_{\mathbf{k}}}{1-\gamma_{\mathbf{k}}}}
$$

which is singular only for $\mathbf{k}=0$. This analysis, for the more general XXZ Hamiltonian with an exchange easy-plane anisotropy $\alpha, 59$ gives

$$
g_{\mathbf{k}}=1-\sqrt{\frac{1+2 \alpha \gamma_{\mathbf{k}}}{1-\gamma_{\mathbf{k}}}} .
$$


In the original (unrotated) reference frame, the Néel state $|N\rangle$ can be written in terms of $|F\rangle=\prod_{i}\left|S_{i}^{x^{\prime}}=s\right\rangle$ by applying the inverse of the unitary transformation (11)

$$
|N\rangle=\hat{\mathcal{U}}|F\rangle=\sum_{x} \mathcal{U}(x)|x\rangle=\sum_{x} \exp \left[+\frac{2 \pi i}{3}\left(\sum_{i \in \mathrm{B}} S_{i}^{z}-\sum_{i \in \mathrm{C}} S_{i}^{z}\right)\right]|x\rangle,
$$

where $|x\rangle$ is an Ising spin configuration specified by assigning the value of $S_{i}^{z}$ (or equivalently of $\left.S_{i}^{z \prime}\right)$ for each site, and $\mathcal{U}(x)=\langle x|\hat{\mathcal{U}}| x\rangle$. Then, introducing a variational parameter $\eta$ scaling the latter potential, for $s=1 / 2$ and in the original spin representation, $\left|\psi_{G}\right\rangle$ assumes the very simple form of Eq. (57), i.e.,

$$
\left|\psi_{G}\right\rangle=\hat{\mathcal{U}}\left|\tilde{\psi}_{G}\right\rangle=\sum_{x} \mathcal{U}(x) \exp \left[\frac{\eta}{2} \sum_{i, j} v(i-j) S_{i}^{z} S_{j}^{z}\right]|x\rangle
$$

where $v(r)=1 / N \sum_{\mathbf{q} \neq 0} e^{-i \mathbf{q} \cdot \mathbf{r}} g_{\mathbf{q}}$ and the summation is restricted only to the Ising configurations with $\sum_{i} S_{i}^{z}=0$ to enforce the projection onto the $S^{z}=0$ subspace. In contrast to the linear spin-wave ground state (48), which does not satisfy the constraint $\left\langle\hat{a}_{i}^{\dagger} \hat{a}_{i}\right\rangle \leq 2 s$, the present variational wavefunction is defined in the correct Hilbert space of the spin operators.

\subsection{Quantum Monte Carlo calculation}

\subsubsection{From Marshal-Peierls to Huse-Elser sign rule}

According to the Marshall theorem (see Sec. 2.1), for the Heisenberg antiferromagnet on the square lattice, as well as on any other bipartite lattice, the classical part of the wavefunction by itself determines exactly the phases of the ground state in the chosen basis. For the triangular case, instead, the exact phases are unknown and the classical part is not enough to fix them correctly. In particular, sfarting from a long-range ordered wavefunction of the form (57), Huse and Elser 45 introduced important three-spin correlation factors in the wavefunction

$$
\hat{T}=\exp \left(i \beta \sum_{\langle i, j, k\rangle} \gamma_{i j k} \hat{S}_{i}^{z} \hat{S}_{j}^{z} \hat{S}_{k}^{z}\right),
$$

defined by the coefficients $\gamma_{i j k}=0, \pm 1$, appropriately chosen so as to preserve the symmetries of the classical Néel state, and by an overall factor $\beta$. In particular the sum in Eq. (64) runs over all distinct triplets of sites $i, j, k$ where both $i$ and $k$ are nearest neighbors of $j$, and $i$ and $k$ are next-nearest neighbors to one another. The sign factor $\gamma_{i j k}=\gamma_{k j i}= \pm 1$ is invariant under rigid translations and rotations in real space by an angle of $2 \pi / 3$ of the three-spin cluster $i, j, k$, but changes sign under rotations by $\pi / 3$ or $\pi$. The resulting wavefunction reads therefore:

$$
\left|\psi_{G}\right\rangle=\sum_{x} \Omega(x) \exp \left(\frac{\gamma}{2} \sum_{i, j} v(i-j) S_{i}^{z} S_{j}^{z}\right)|x\rangle,
$$



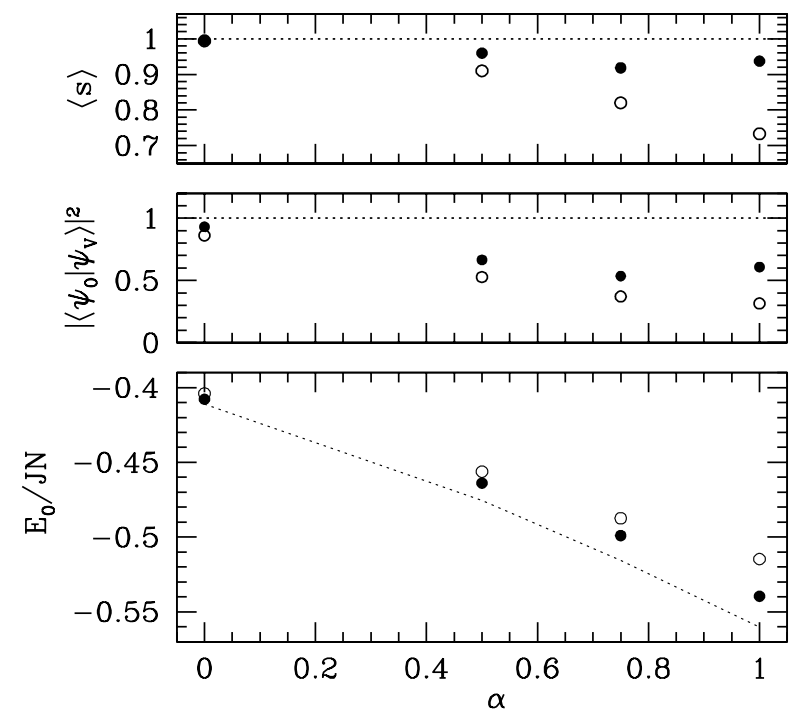

Figure 6: Average sign, overlap square and ground-state energy per site obtained for $N=36$ using the variational wavefunction of Eq. (65), with (full dots) and without (empty dots) the triplet term of Eq. (64), as a function of the easy-plane anisotropy $\alpha$. The calculations were performed by summing exactly over all the configurations and the dotted line connects the exact results.

with a phase factor given by

$$
\Omega(x)=T(x) \exp \left[+\frac{2 \pi i}{3}\left(\sum_{i \in \mathrm{B}} S_{i}^{z}-\sum_{i \in \mathrm{C}} S_{i}^{z}\right)\right]
$$

where $T(x)=\langle x|\hat{T}| x\rangle$. Finally, since the Hamiltonian is real, a better variational wavefunction on a finite size is obtained by taking the real part of Eq. (65).

Although the three-body correlations of Eq. (64) do not provide the exact answer, they allow us to adjust the signs of the wavefunction in a nontrivial way without changing the underlying classical Néel order. In this respect it is useful to define an average sign of the variational wavefunction relative to the normalized exact ground state $\left|\psi_{0}\right\rangle$ as

$$
\langle s\rangle=\sum_{x}\left|\psi_{0}(x)\right|^{2} \operatorname{sgn}\left[\psi_{G}(x) \psi_{0}(x)\right],
$$

with $\psi(x)=\langle x \mid \psi\rangle$. We have compared the variational calculation with the exact ground state obtained by ED on the $N=36$ cluster. For completeness we have considered the more general XXZ Hamiltonian with the exchange easy-plane anisotropy $\alpha$, ranging from the XY case $(\alpha=0)$ to the standard spin-isotropic case $(\alpha=1)$. As shown in Fig. 6, the introduction of the three-body correlations of 


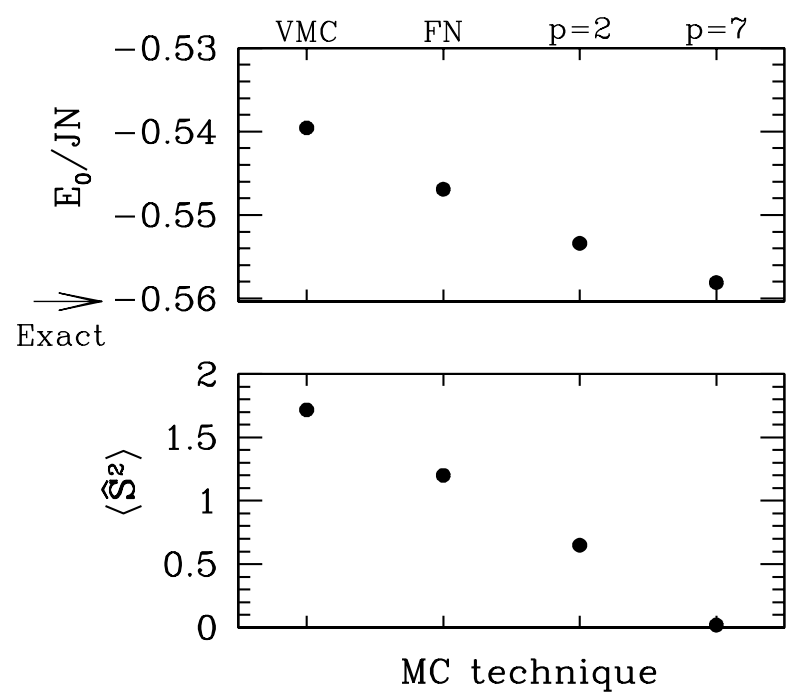

Figure 7: Variational estimate (VMC) and mixed averages (FN, GFMCSR) of the ground-state energy per site $E_{0} / J N$ and of the total spin square $\left\langle\hat{\mathbf{S}}^{2}\right\rangle$ for $N=36$. GFMCSR data are obtained using the short-range correlation functions generated by $\hat{\mathcal{H}}(p=2)$, and $\hat{\mathcal{H}}^{2}(p=7)$ as explained in the text.

Eq. (64), although not providing the exact answer, improves the overlap square of the variational wavefunction on the true ground state and the accuracy of the variational estimate of the ground-state energy as well. In particular the average sign $\langle s\rangle$ is very much improved by the triplet term, particularly in the spin-isotropic limit $\alpha \rightarrow 1$. This is crucial when the variational wavefunction is used for importance sampling within the modifications of the GFMC technique developed to handle the sign problem instability, like the FN and GFMCSR techniques. In the following the wavefunction (65) will be used as the guiding wavefunction in our quantum Monte Carlo calculations.

\subsubsection{The reconfiguration scheme}

In order to study the ground-state properties we have used the GFMCSR quantum Monte Carlo technique, which allows one to release the FN approximation, in an approximate but controlled way. This systematic improvement introduced by the GFMCSR on the accuracy of the ground-state properties, is illustrated in Fig. I, where we display a comparison between the estimates of the ground-state energy per site and of total spin square, for the $N=36$, obtained with the stochastic sampling of the variational wavefunction (65), the FN and the GFMCSR techniques. As explained in Refs. 17.18 , in the appropriate limit of large number of walkers and high frequency of SR, the residual bias introduced by the GFMCSR depends only on the number $p$ of operators used to constrain the GFMC Markov process, 

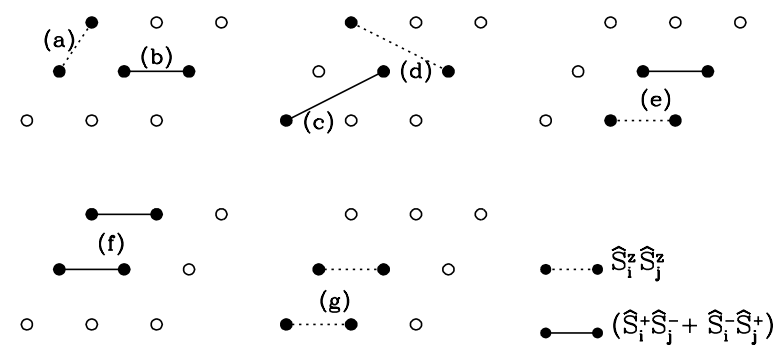

Figure 8: $\quad$ Short range spin correlation functions generated by $\hat{\mathcal{H}}(\mathrm{a}, \mathrm{b})$ and $\hat{\mathcal{H}}^{2}$ (c-g).

allowing simulations without numerical instabilities. In principle the exact answer can be obtained, within statistical errors, provided $p$ equals the huge Hilbert space dimension. In practice it is necessary to work with small $p$ and an accurate selection of physically relevant operators is therefore crucial. As can be easily expected, the short-range correlation functions $\hat{S}_{i}^{z} \hat{S}_{j}^{z}$ and $\left(\hat{S}_{i}^{+} \hat{S}_{j}^{-}+\hat{S}_{i}^{-} \hat{S}_{j}^{+}\right)$contained in the Hamiltonian $(p=2)$ give a sizable improvement of the FN ground-state energy when they are included in the SR procedure. In order to be systematic, we have included in the SR also the short-range correlations generated by $\hat{\mathcal{H}}^{2}(p=7)$, averaged over all spatial symmetries commuting with the Hamiltonian. These local correlations (see Fig. 8) are particularly important to obtain quite accurate and reliable estimates not only of the ground-state energy but also of the mixed average 27.18 of the total spin square $\hat{\mathbf{S}}^{2}$. In particular it is interesting that, starting from a variational wavefunction with no definite spin, the spin rotational invariance of the finite-size ground state is systematically recovered by means of the GFMCSR technique (see lower panel of Fig. (7).

Having obtained an estimate for the ground-state energy, at least an order of magnitude more accurate than our best variational guess, it appears possible to obtain physical features, such as a gap in the spin spectrum, that are not present at the variational level. For instance in the frustrated $J_{1}-J_{2}$ Heisenberg model (see Sec. 4.2), with the same technique and a similar accuracy, a gap in the spin spectrum is found in the thermodynamic limit, starting with a similar ordered and therefore gapless variational wavefunction.

\subsubsection{Ground-state energy and spin gap}

In presence of Néel long-range order, being the magnon dispersion relation linear in the wavevector $\mathbf{k}$, the leading finite-size correction to the ground-state energy per site is $\mathcal{O}\left(N^{-3 / 2}\right): 13$ this is clearly shown by the behavior of the finite-size spin-wave results in Fig. 9. In the same figure the size scaling of the estimates of the groundstate energy per site obtained with the VMC, the FN and the GFMCSR $(p=7)$ techniques is also reported. The predicted size scaling, fulfilled of course by the 


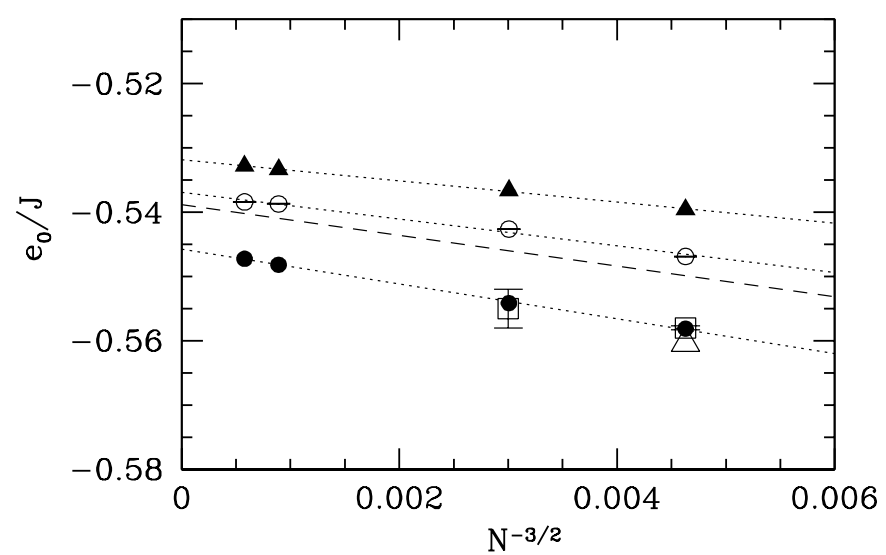

Figure 9: Ground-state energy per site $e_{0}=E_{0} / N$, in unit of $J$, as a function of the system size, obtained with VMC (full triangles), FN (empty dots) and GFMCSR with $p=7$ (full dots) techniques. Spin-wave size scaling is assumed and shortdashed lines are linear fits against $1 / N^{3 / 2}$. The long-dashed line is the linear spinwave prediction, the empty triangle is the $N=36 \mathrm{ED}$ result and the empty squares are data taken from Ref. 36.

variational wavefunction (65), is also preserved within the $\mathrm{FN}$ and the more accurate GFMCSR technique, thus providing a first clue on the existence of long-range Néel order in the thermodynamic ground state of the model. The quality of our results is similar to the variational one obtained by Sindzingre and co-workers 36 using a long-range ordered RVB wavefunction (Sec. 2.4). The latter approach is almost exact for small lattices, but the sign problem is already present at the variational level, and the calculation has not been extended to high statistical accuracy or to $N>48$. Our best estimate is that in the thermodynamic limit the ground-state energy per site is $e_{0}=-0.5458 \pm 0.0001$ in unit of the exchange coupling.

In the isotropic triangular antiferromagnet, the gap to the first spin excitation is rather small. Furthermore, for the particular choice of the guiding wavefunction (65), the translational symmetry of the Hamiltonian is preserved only if projected onto subspaces with total $S^{z}$ multiple of three. Then, we have studied the gap to the spin $S=3$ excitation as a function of the system size. Technically, within our numerical framework, such a spin gap can be evaluated by performing two simulations in the $S^{z}=0$ and $S^{z}=3$ subspaces. This can be easily done by restricting the sampling to the configurations $|x\rangle$ in Eq. (65) with the desired value of $S^{z}$. In this case the potential $v(r)$ used was the same in both subspaces and the variational parameter $\eta$ was found by optimizing the energy in the $S^{z}=0$ subspace.

As it is shown in Fig. 10, for the lattice sizes for which a comparison with ED data is possible, the spin gap estimated with the GFMCSR technique is nearly exact. The importance of extending the numerical investigation to clusters large 


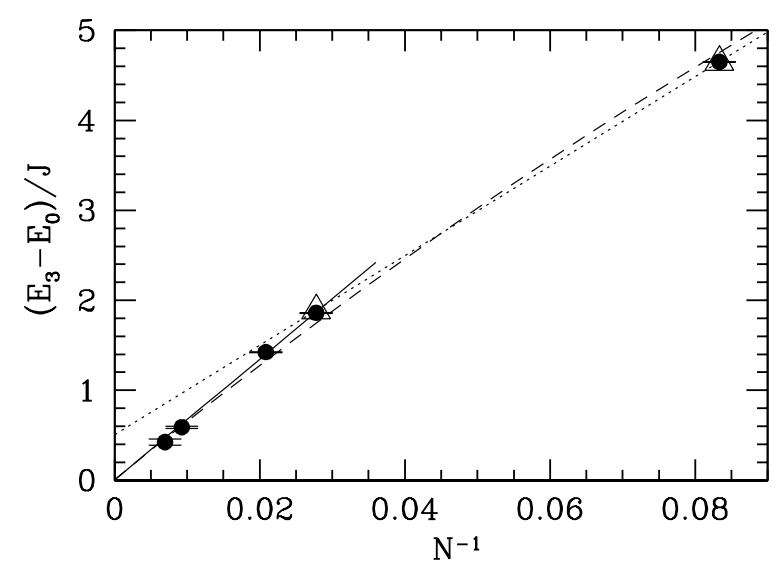

Figure 10: Size scaling of the spin gap to the $S=3$ excitation obtained with the GFMCSR $(p=7)$ technique (full dots). Empty triangles are the ED results, the long-dashed line is the linear spin-wave prediction, the dotted line is the linear extrapolation of the $N=12,36$ exact results and the solid line is the least-squares fit of the GFMCSR data for $N \geq 36$.

enough to allow a more reliable extrapolation is particularly evident in the same figure, in which the $N=12$ and 36 exact data extrapolate linearly to a large finite value. This behavior, is certainly a finite-size effect and it is corrected by the GFMCSR data for $N \geq 48$, suggesting, strongly, a gapless excitation spectrum $\left[\left(E_{3}-E_{0}\right) / J=0.002 \pm 0.01\right]$.

\subsubsection{Staggered magnetization}

The GFMC allows us to obtain a very high statistical accuracy on the groundstate energy, but does not allow us to compute directly ground-state expectation values $\left\langle\psi_{0}|\hat{O}| \psi_{0}\right\rangle$. A straightforward way to calculate such expectation values is to use the Hellmann-Feynman theorem. In fact, if the Hamiltonian is perturbed with a term $-\lambda \hat{O}$ the first order correction to the ground-state energy is, by standard perturbation theory,

$$
E(\lambda)=E_{0}-\lambda\left\langle\psi_{0}|\hat{O}| \psi_{0}\right\rangle
$$

As a consequence it is possible to evaluate the ground-state expectation value $\left\langle\psi_{0}|\hat{O}| \psi_{0}\right\rangle=-d E(\lambda) /\left.d \lambda\right|_{\lambda=0}$ estimating the limit

$$
\left\langle\psi_{0}|\hat{O}| \psi_{0}\right\rangle=-\lim _{\lambda \rightarrow 0} \frac{E(\lambda)-E_{0}}{\lambda}
$$

with few computations at different small $\lambda$ 's.

A further complication for nonexact calculations like the FN or GFMCSR, is that if the off-diagonal matrix elements $\left\langle x^{\prime}|\hat{O}| x\right\rangle$ of the operator $\hat{O}$ (in the chosen 


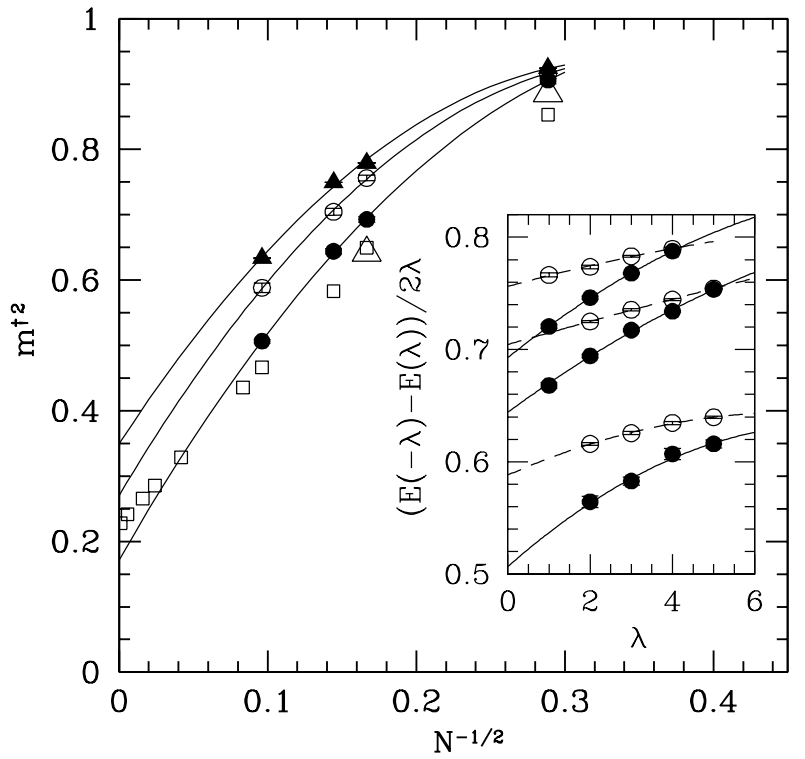

Figure 11: Size scaling of the order parameter: VMC (full triangles), FN (empty dots), GFMCSR (full dots), exact data (empty triangles) and finite-size linear spin wave theory 57 (empty squares). The inset displays the $\lambda \rightarrow 0$ extrapolation for $N>12$. Lines are quadratic fits in all the plots.

basis) have signs which are opposite to those of the product $\psi_{G}\left(x^{\prime}\right) \psi_{G}(x)$, they cannot be handled exactly within the FN because the addition of such a perturbation to the Hamiltonian changes the nodal surface of the guiding wavefunction. In that case, in fact, the effective FN Hamiltonian associated to the unperturbed Hamiltonian is also affected by the presence of the field and this leads naturally to the breakdown of Eq. (68). A way out of this difficulty if to split the operator $\hat{O}$ into three contributions: $\hat{O}=\hat{D}+\hat{O}^{+}+\hat{O}^{-}$, where $\hat{O}^{+}\left(\hat{O}^{-}\right)$is the operator with the same off-diagonal matrix elements of $\hat{O}$ when they have the same (opposite) signs of $\psi_{G}\left(x^{\prime}\right) \psi_{G}(x)$, and zero otherwise, whereas $\hat{D}$ is the diagonal part of $\hat{O}$. Then, we can add to the Hamiltonian a contribution that does not change the nodes: $\hat{\mathcal{H}}(\lambda)=\hat{\mathcal{H}}-\lambda\left(\hat{D}+2 \hat{O}^{+}\right)$for $\lambda>0$ and $\hat{\mathcal{H}}(\lambda)=\hat{\mathcal{H}}-\lambda\left(\hat{D}+2 \hat{O}^{-}\right)$for $\lambda<0$. Then the expectation value of the operator $\hat{O}$ can be written as

$$
\left\langle\psi_{0}|\hat{O}| \psi_{0}\right\rangle=\lim _{\lambda \rightarrow 0} \frac{E(-\lambda)-E(\lambda)}{2 \lambda} .
$$

With this method, using the FN and GFMCSR techniques, we have calculated the order parameter

$$
m^{\dagger 2}=36 \frac{\mathcal{M}^{2}}{N(N+6)}
$$


where $\mathcal{M}^{2}$ is the sublattice magnetization squared 13 Our results are plotted in Fig. 11. For the order parameter the inclusion of many short-range correlations in the SR is not very important. Then, in order to minimize the numerical effort, we have chosen to put in the SR conditions the first four correlation functions shown in Fig. 9, the order parameter itself and $\hat{\mathbf{S}}^{2}$. While the FN data extrapolate to a value not much lower than the variational result, the GFMCSR calculation provides a much more reliable estimate of the order parameter with no apparent loss of accuracy with increasing sizes. In this way we obtain for $\hat{m}^{\dagger}$ a value well below the linear and the second order (which has actually a positive correctionE2) spinwave predictions. Our best estimate is that in the thermodynamic limit the order parameter $m^{\dagger}=0.41 \pm 0.02$ is reduced by about $59 \%$ from its classical value. This is partially in agreement with the conclusions of the finite-temperature calculations 4 suggesting a ground state with asmall but nonzero long-range antiferromagnetic order and with series expansions 46 indicating the triangular antiferromagnet to be likely ordered but close to a critical point. In our simulation, however, which to our knowledge represents a first attempt to perform a systematic size-scaling analysis of the order parameter, the value of $\hat{m}^{\dagger}$ remains sizable and finite, consistent with a gapless spectrum.

\section{The $J_{1}-J_{2}$ Heisenberg Model}

In the triangular Heisenberg antiferromagnet, frustration is induced by the geometry of the lattice. The other possible origin of the frustration comes from competing interactions as in the so-called $J_{1}-J_{2}$ Heisenberg model on the square lattice defined in Eq. (2) of the Introduction.

In the last few years seyeral studies - including exact diagonalizations of small clusters 22, 60 spin-wave $106,61,62$ and Schwinger-boson 63 calculations, series $64,65,66$ and

large- $N$ 67 expansions - have provided some evidence for the absence of Néel order in the ground state of the spin- $1 / 2 J_{1}-J_{2}$ Heisenberg model for $0.38<J_{2} / J_{1}<0.6$. However, due to the difficulties related to the sign problem, a systematic size-scaling of the spin gap has been lacking for many years, and no definite conclusion on the nature of the non-magnetic phase has been drawn yet. In particular, an open question is whether the ground state in the quantum disordered phase is a RVB spin liquid with no broken symmetry 68 or if it breaks some crystal symmetries by dimerizing in some special pattern (Sec. 2.4).

In this section, we will address the problem of the quantum phase-transition to a non-magnetic ground state driven by frustration in the spin-1/2 $J_{1}-J_{2}$ Heisenberg model by means of the finite-size spin-wave theory, ED and GFMC techniques.

\subsection{Finite-size spin-wave results}

The finite-size spin-wave theory for the spin-s $J_{1}-J_{2}$ Heisenberg model can be derived along the same lines followed for the triangular Heisenberg antiferromagnet in Sec. 3.1. In this section we will only show the main results for $J_{2} / J_{1}<0.5$, 
assuming the two sublattice classical Néel order [Fig. 22 (a)] in the $x y$-plane.

Applying the unitary transformation (6) which rotates the spin quantization axis by a angle $\pi$ about the $z$-axis on one of the two sublattices, setting the order parameter along the $x$-axis, and using the Holstein-Primakoff transformation for spin operators at the leading order in $1 / s$, the Fourier transformed spin-wave Hamiltonian results as in Eq. (40) with $E_{c l}=-z J s^{2} N(1-\beta) / 2, z=4, \beta=J_{2} / J_{1}$,

$$
A_{\mathbf{k}}=1+\beta\left(\delta_{\mathbf{k}}-1\right), B_{\mathbf{k}}=-\gamma_{\mathbf{k}},
$$

$\gamma_{\mathbf{k}}=\left(\cos k_{x}+\cos k_{y}\right) / 2$, and $\delta_{\mathbf{k}}=\cos k_{x} \cos k_{y}$.

Similarly to the triangular lattice case, the singular Goldstone modes $[\mathbf{k}=\mathbf{0}$ and $\mathbf{k}=(\pi, \pi)$ ] cannot be diagonalized by means of the Bogoliubov transformation but can be recombined to give the total spin squared $\hat{\mathbf{S}}^{2}$ at the leading order in $1 / s$ :

$$
\hat{\mathcal{H}}_{S M}=-J_{1} s z A_{\mathbf{0}}+J_{1} z \frac{A_{\mathbf{0}}}{N}\left[\left(S^{x}\right)^{2}+\left(S^{y}\right)^{2}+\left(S^{z}\right)^{2}\right] .
$$

As seen in Sec. 3.1 this term, being $A_{0}$ positive definite, favors a singlet ground state and implies the Lieb-Mattis property, which has been demonstrated only for bipartite Hamiltonians, but nonetheless can be verified numerically on finite sizes for the $J_{1}-J_{2}$ Heisenberg model.

As in the triangular case, the above analysis allows one to derive a variational wavefunction which is both accurate and easily computable in a quantum Monte Carlo algorithm. Here we will not repeat the derivation, which follows very closely the one for the triangular antiferromagnet, and leads to the following result for $s=1 / 2$ :

$$
\left|\psi_{G}\right\rangle=\sum_{x} S_{M}(x) \exp \left[\frac{\eta}{2} \sum_{i, j} v(i-j) S_{i}^{z} S_{j}^{z}\right]|x\rangle,
$$

where $v(r)=1 / N \sum_{\mathbf{q} \neq 0} e^{-i \mathbf{k} \cdot \mathbf{r}} g_{\mathbf{k}}$ with

$$
g_{\mathbf{k}}=\frac{v_{\mathbf{k}}}{v_{\mathbf{k}}-u_{\mathbf{k}}}=1-\sqrt{\frac{1-\beta\left(1-\delta_{\mathbf{k}}\right)+\gamma_{\mathbf{k}}}{1-\beta\left(1-\delta_{\mathbf{k}}\right)-\gamma_{\mathbf{k}}}} ;
$$

$|x\rangle$ is an Ising spin configuration specified by assigning the value of $S_{i}^{z}$ for each site and $S_{M}(x)=(-1)^{N_{\uparrow}(x)}$ is the Marshall sign (Sec. 2.1), depending on the number $N_{\uparrow}(x)$ of spin up on one of the two sublattices. The summation is restricted only to the Ising configurations with $\sum_{i} S_{i}^{z}=0$ in order to enforce the projection onto the $S^{z}=0$ subspace. In the following we will use the latter variational wavefunction as the starting point for more refined quantum Monte Carlo calculations. In particular for $J_{2} / J_{1}=0.5$ we have chosen to work with $\beta=0.4$ in Eq. (75). The possibility to restrict to any total spin projection $S^{z}=\sum_{i} S_{i}^{z}$ allows one to evaluate the spin gap by performing two simulations for $S^{z}=0$ and $S^{z}=1$. As in the triangular case, the potential $v(r)$ used was the same in both subspaces and the variational parameter $\eta$ was found by optimizing the energy in the $S^{z}=0$ subspace. The latter 
Table 2. Estimates of the ground-state energy per site and their relative accuracy (in brackets) for $N=36$ and various values of the $J_{2} / J_{1}$ ratio. VMC: variational Monte Carlo. VMCLS: variational Monte Carlo with LS wavefunction. SRLS: GFMCSR with LS wavefunction and $p=8$ (see text).

\begin{tabular}{ccccc}
\hline$J_{2} / J_{1}$ & VMC & VMCLS & SRLS & Exact \\
\hline 0.0 & $-0.6695(1.4 \%)$ & $-0.6756(0.5 \%)$ & $-0.6789(0.00 \%)$ & -0.67887 \\
0.1 & $-0.6284(1.5 \%)$ & $-0.6349(0.5 \%)$ & $-0.6379(0.03 \%)$ & -0.63810 \\
0.2 & $-0.5884(1.8 \%)$ & $-0.5952(0.6 \%)$ & $-0.5988(0.04 \%)$ & -0.59905 \\
0.3 & $-0.5495(2.3 \%)$ & $-0.5574(0.9 \%)$ & $-0.5619(0.10 \%)$ & -0.56246 \\
0.4 & $-0.5120(3.3 \%)$ & $-0.5237(1.1 \%)$ & $-0.5289(0.16 \%)$ & -0.52974 \\
0.5 & $-0.4783(5.1 \%)$ & $-0.4916(2.4 \%)$ & $-0.5022(0.32 \%)$ & -0.50381 \\
\hline
\end{tabular}

spin-wave variational wavefunction (see Tab. 2) provides a rather good estimate of the ground-state energy for $J_{2} / J_{1} \leq 0.3$. Instead such accuracy abruptly decreases instead in the regime of strong frustration, suggesting a change in the nature of the ground state.

Within the finite-size spin-wave theory, we can also gain information about the low-lying excited states. As shown in Sec. 3.1.2, in order to stabilize a low-energy total spin excitation $S$ a magnetic field $h$ in the $z$-direction must be added to the spin Hamiltonian. Keeping into account that, for small fields, the classical minimum energy configuration is the Néel order canted by an angle $\theta$ along the direction of $h$ (with $\sin \theta=h / 2 J_{1} z$ ), the finite-size spin-wave expansion is straightforward and leads to a linearized Hamiltonian as in Eq. (40) with the following field-dependent coefficients

$$
A_{\mathbf{k}}^{h}=1+\beta\left(\delta_{\mathbf{k}}-1\right)+\gamma_{\mathbf{k}}\left(\frac{h}{2 J_{1} z}\right)^{2}, B_{\mathbf{k}}^{h}=-\gamma_{\mathbf{k}}\left[1-\left(\frac{h}{2 J_{1} z}\right)^{2}\right],
$$

and with a singular part given by

$$
\mathcal{H}_{S M}=-\frac{J_{1} s z}{2} A_{\mathbf{0}}^{h}+\frac{J_{1} z}{N}\left(S^{z}-N s \sin \theta\right)^{2}
$$

favoring a value of $S^{z}$ (in the original spin representation) consistent with the applied field, at the classical level.

The total spin $S=N\left\langle S_{i}^{z}\right\rangle$ of the excitation can be related to the magnetic field $h$ by means of the Hellmann-Feynman theorem

$$
\left\langle S_{i}^{z}\right\rangle=-\frac{1}{N s} \frac{\partial}{\partial h} E(h)=s \frac{h}{2 J_{1} z}\left[1+\frac{1}{2 N s} \sum_{\mathbf{k} \neq 0} \gamma_{\mathbf{k}} \sqrt{\frac{A_{\mathbf{k}}^{h}+B_{\mathbf{k}}^{h}}{A_{\mathbf{k}}^{h}-B_{\mathbf{k}}^{h}}}\right]
$$

where

$$
E(h)=E_{c l}-(s h)^{2} \frac{N}{4 J_{1} z}-\frac{J_{1} s z}{2}\left[N(1-\beta)-\sum_{\mathbf{k}} \epsilon_{\mathbf{k}}^{h}\right]
$$




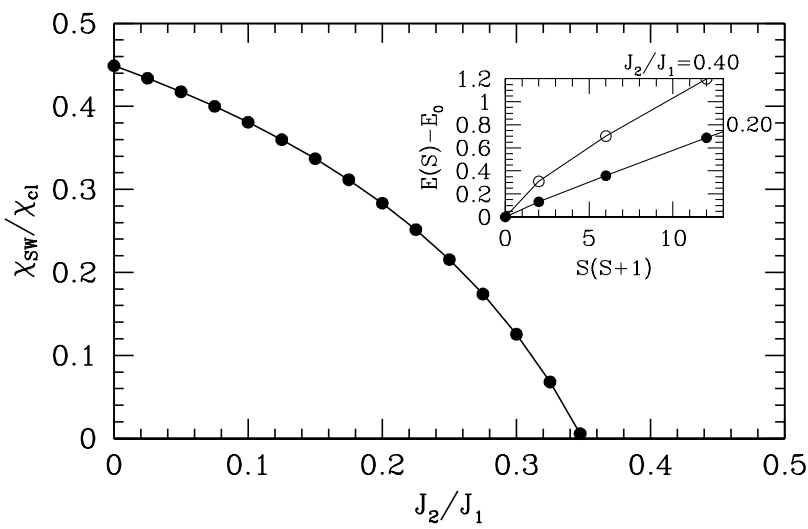

Figure 12: Spin-wave uniform susceptibility as a function of $J_{2} / J_{1}$ for $s=1 / 2$. The inset displays the low-energy spin-wave spectrum for $N=144$ for two values of the $J_{2} / J_{1}$ ratio as a function of $|\mathbf{S}|^{2}=S(S+1)$.

and $\epsilon_{\mathbf{k}}^{h}=\sqrt{\left(A_{\mathbf{k}}^{h}\right)^{2}-\left(B_{\mathbf{k}}^{h}\right)^{2}}$. The final step in order to evaluate the energy spectrum $E(S)$ is to perform a Legendre transformation $E(S)=E(h)+h s S$.

Finally, the spin-wave uniform susceptibility,

$$
\chi_{\mathrm{SW}}=-1 / N \partial^{2} E(h) /\left.\partial h^{2}\right|_{h=0},
$$

is, at the leading order in $1 / s$,

$$
\chi_{\mathrm{SW}} / \chi_{c l}=1+\frac{1}{2 N s} \sum_{\mathbf{k} \neq 0} \gamma_{\mathbf{k}} \sqrt{\frac{A_{\mathbf{k}}+B_{\mathbf{k}}}{A_{\mathbf{k}}-B_{\mathbf{k}}}} .
$$

where $\chi_{c l}=1 / 2 J_{1} z$.

\subsection{Transition to a quantum disordered state induced by frustration}

\subsubsection{Spin-wave susceptibilities and low-energy spectra}

For the unfrustrated Heisenberg model, even for $s=1 / 2$, the spin-wave predictions are very accurate as far as the energy, the order parameter and the spin uniform susceptibility are concerned.10 32 Turning on $J_{2}$ the model is increasingly frustrated and one can expect the spin-wave theory to remain accurate only in the region where the nature of the order parameter is the same as in the classical case $(S \rightarrow \infty)$. Within this analytical approach we can therefore detect a non-magnetic phase by looking for the breakdown of the spin-wave expansion.

As pointed out by Zhong and Sorella, 10 for moderate frustration $\left(J_{2} / J_{1}<0.2\right)$ the linear spin-wave predictions on finite sizes are quite accurate for both the energy and the antiferromagnetic order parameter. Moreover, in this regime, the second 

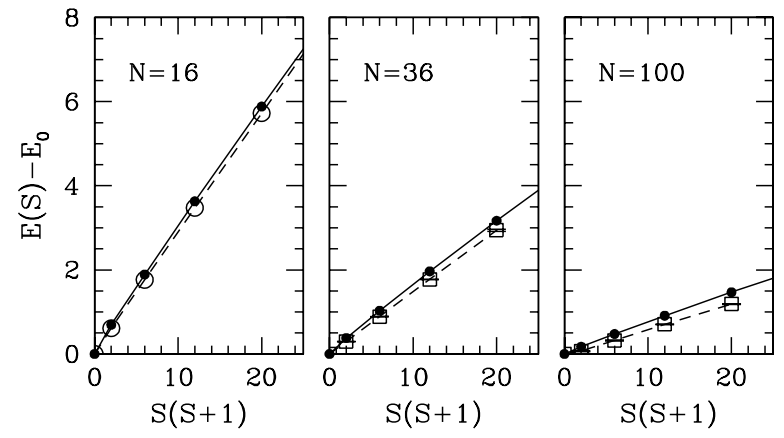

Figure 13: Low-energy excitation spectra as a function of $|\mathbf{S}|^{2}=S(S+1)$ for $J_{2} / J_{1}=0.2, s=1 / 2$ and $N=16,36,100$ : spin-wave (full circles and continuous line), exact (empty circles and dashed lines), GFMCSR with $p=8$ (empty squares and dashed lines).

order correction 10 leads to an almost exact result. For large $J_{2} / J_{1}$, instead, the second order term does not improve the first order estimate and a possible breakdown of the spin-wave expansion may occur even well below the classical transition point $J_{2} / J_{1}=0.5$. In particular at the leading order in $1 / s$ and for $s=1 / 2$ the order parameter vanishes at a critical value $\left(J_{2} / J_{1}\right)_{c} \simeq 0.38$. 10.61

Analogously, a breakdown of the spin-wave expansion can be evidenced from the vanishing of the uniform susceptibility, which is always finite when there is longrange Néel order in the thermodynamic limit and vanishes instead in presence of a finite triplet gap (see Sec. 2.2). As it is shown in Fig. 12, the classical uniform susceptibility is strongly renormalized by the quantum fluctuations for $s=1 / 2$ at the spin-wave level (80). As expected, increasing the frustration such reduction is enhanced and leads eventually to the vanishing of the susceptibility for $J_{2} / J_{1} \simeq 0.35$. The structure of the finite-size spin-wave excitation spectrum below and above this critical point is very different (see the inset of Fig. 12) with an evident breakdown of the quantum top law (30), as well as of the spin-wave approximation scheme, in the non-magnetic phase. Below the critical point, instead, the spin-wave theory reproduces remarkably well the exact and GFMCSR results for the low-energy part of the spectrum in the whole range of sizes (see Fig. 13). Furthermore, as already observed for the triangular antiferromagnet, increasing the size, the slope of $E(S)$ vs $S(S+1)$ decreases and gives rise to the collapse of a macroscopic number of states with different $S$ on the ground state as $N \rightarrow \infty$ : i.e., a ground state with a broken $\mathrm{SU}(2)$ symmetry.

\subsubsection{Size-scaling of the spin gap}

The spin-wave prediction for the occurrence of a non-magnetic region in the phase diagram of the $J_{1}-J_{2}$ Heisenberg model is confirmed by our results for the 


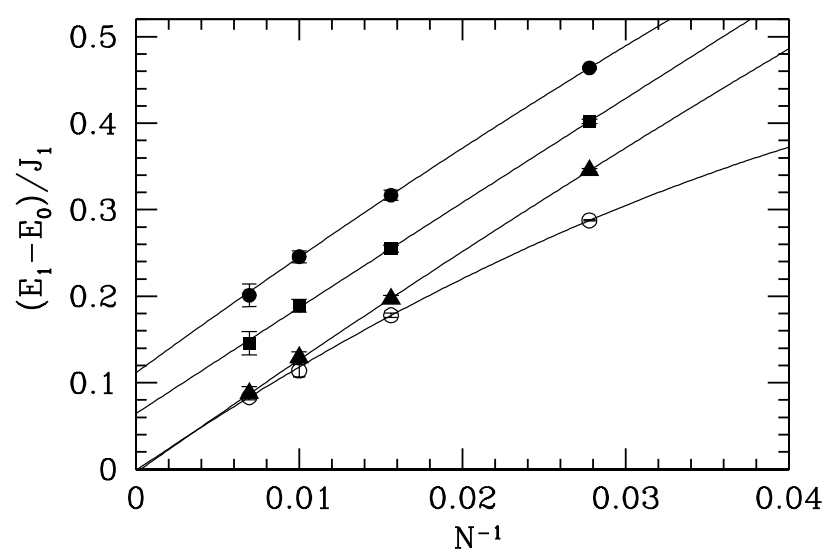

Figure 14: Size scaling of the energy gap to the first $S=1$ spin excitation obtained with the GFMCSR technique for $J_{2} / J_{1}=0.38$ (full triangles), 0.45 (full squares) and 0.50 (full circles). Data for the unfrustrated $\left(J_{2}=0\right)$ Heisenberg model taken from Ref. 69, are also shown for comparison (empty circles). Lines are weighted quadratic fits of the data.

spin triplet gap obtained using the GFMCSR. The latter calculation, which extends the recent one by Sorella, 17 has been performed using (74) as guiding wavefunction and including in the SR conditions the energy, all $\hat{S}_{i}^{z} \hat{S}_{j}^{z}$ independent by symmetry, and the antiferromagnetic order parameter. The latter, though not improving the accuracy of the calculation, allows a very stable and reliable simulation for large $p$. The new results, extended up to $N=144$, confirm the previous finding, 17 of a finite spin gap in the thermodynamic limit for $J_{2} / J_{1}>0.40$ (Fig. 14). Remarkably, these results are not an artifact of the chosen guiding wavefunction: in fact, unlike the FN approximation, the GFMCSR is able to detect a finite gap in the thermodynamic limit by starting from a spin-wave like wavefunction (74) which is Néel ordered and therefore gapless. This behavior is very different from the one observed in the case of the $s=1 / 2$ Heisenberg antiferromagnet on the triangular lattice (see Fig. 10) where, with the same numerical scheme, a similar guiding wavefunction and a comparable accuracy we obtained a gapless excitation spectrum. Therefore the existence of a gapped phase in the regime of strong frustration is likely to be a genuine feature of the $J_{1}-J_{2}$ Heisenberg model.

\subsection{The nature of the non-magnetic phase}

In principle either a RVB crystal, with some broken spatial symmetry, or a homogeneous spin liquid is compatible with a triplet gap in the excitation spectrum. Among the dimerized phases proposed in the literature, the so-called columnar and plaquette RVB are the states which are the most likely candidates. These kind of

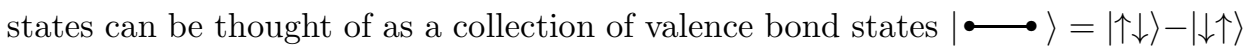




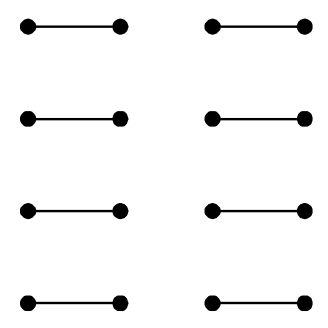

(a)

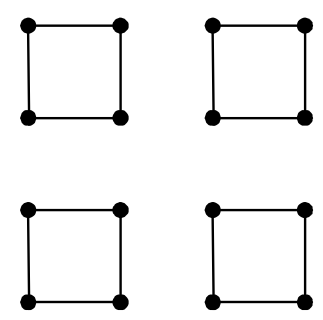

(b)

Figure 15: Columnar (a) and plaquette (b) RVB states.

between neighboring sites arranged in the patterns shown in Fig. 15, where the plaquettes in (b) are the following rotationally invariant superpositions:

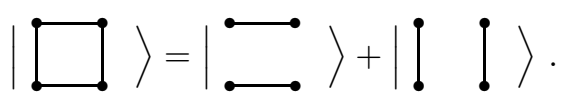

Both the columnar and the plaquette states break the translation invariance along the $x$ and $y$ directions, but only the latter preserves the symmetry of interchange of the two axes.

Read and Sachdev67 with a field-theoretic large- $N$ expansion, were the first to conjecture that quantum fluctuations and a next-nearest-neighbor frustrating interaction could drive the ground state of the square lattice antiferromagnet into a columnar RVB state and series expansion studies 64.63 have supported over the years this prediction. Recently, Kotov and co-worker 70 with a study that combines an analytic effective Hamiltonian approach, extended dimer expansions and exact diagonalizations have presented a body of evidences that has been interpreted as supporting the columnar scenario. Finally, using the GFMCSR with a Density Matrix Renormalization Group guiding wavefunction, du Croo de Jongh and coworker 71 have proposed a ground state with intermediate properties between the plaquette and columnar RVB.

\subsubsection{The method of generalized susceptibilities}

In order to better characterize the nature of the ground state in the gapped phase, we have checked the occurrence of some kind of crystalline order, by calculating the response of the system to operators breaking the most important lattice symmetries. As suggested in Refs. 65.66.62, and also shown in Sec. 2.2, the occurence of some kind of crystalline order in the thermodynamic ground state can be checked by adding to the Hamiltonian (2) a term $-\delta \hat{O}$, where $\hat{O}$ is an operator that breaks some symmetry of $\hat{\mathcal{H}}$. In fact, if true long-range order exists in the thermodynamic ground state, the finite-size susceptibility $\chi_{O}=\langle\hat{O}\rangle_{\delta} / N \delta$ has to diverge with the system size and, in particular, it is bounded from below by the system volume 

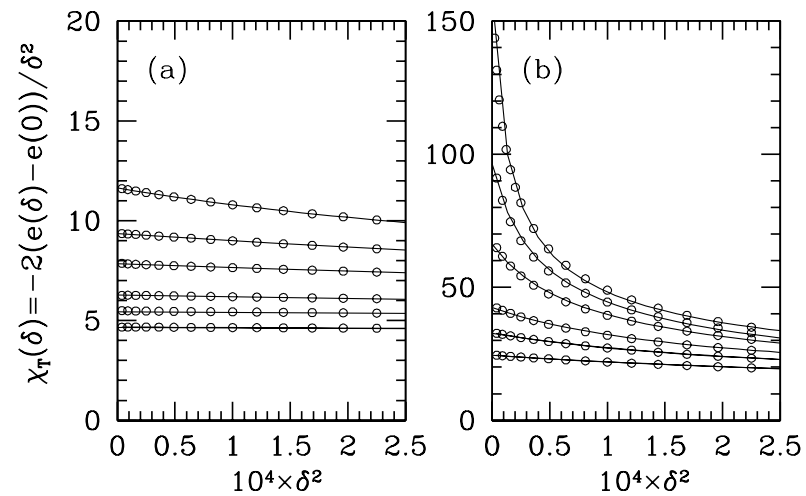

Figure 16: Exact results for the $J_{1}-J_{2}$ chain: $\chi_{\mathrm{T}}(\delta)$ associated to the operator $\hat{O}_{\mathrm{T}}$ (breaking the translational invariance) for $J_{2} / J_{1}=0.2(\mathrm{a})$ and $J_{2} / J_{1}=0.4(\mathrm{~b})$. Data are shown for $N=12,14,16,20,24,30$ for increasing values of $\chi_{\mathrm{T}}(\delta)$. Lines are guides for the eye.

squared [Eq. (27)]. Thus susceptibilities are in principle a very sensitive tool much more than the square of the order parameter - for detecting the occurrence of long-range order.

Within a numerical technique, the susceptibility $\chi_{O}=d^{2} e(\delta) /\left.d \delta^{2}\right|_{\delta=0}$ can be calculated with only energy measurements by computing the ground-state energy per site in presence of the perturbation for few values of $\delta$ and by estimating numerically the limit

$$
\chi_{O}=\lim _{\delta \rightarrow 0} \chi_{O}(\delta)=-\frac{2\left(e(\delta)-e_{0}\right)}{\delta^{2}} .
$$

As we have tested in the one dimensional $J_{1}-J_{2}$ model, the numerical study of long-range order by means of $\chi(\delta)$ is very effective and reliable. Here a quantum critical point at $J_{2} / J_{1} \simeq 0.2412$ separating a gapless spin-fluid phase from a gapped dimerized ground state (which is two-fold degenerate and adiabatically connected to the Majumdar-Ghosh exact solution for $J_{2} / J_{1}=0.5$ ) is rather well accepted. 73.74 .75 As shown in Fig. 16, the response of the system to the perturbation $\delta \hat{O}_{T}$, with

$$
\hat{O}_{\mathrm{T}}=\sum_{j} e^{i k j} \hat{\mathbf{S}}_{j} \cdot \hat{\mathbf{S}}_{j+x}
$$

breaking the translation invariance with momentum $k=\pi$, is very different below and above the dimer-fluid transition point. However it is extremely important to perform very accurate calculations at small $\delta$ to detect the divergence of the susceptibilities for large system sizes.

\subsubsection{Stability of Plaquette vs Columnar RVB}




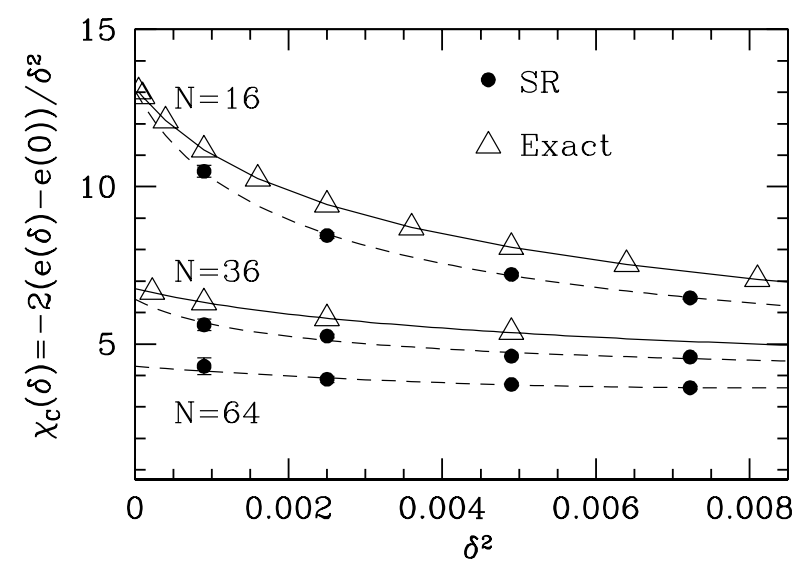

Figure 17: Exact and GFMCSR calculation of $\chi_{\mathrm{C}}(\delta)$ associated to $\hat{O}_{\mathrm{C}}$ (columnar dimerization) for $J_{2} / J_{1}=0.5$. Lines are guides for the eye.

As also suggested in a recent paper by Singh and co-workers, 60 the appearance of a columnar state can be probed by using as order parameter the operator

$$
\hat{O}_{\mathrm{C}}=\sum_{i}\left(\hat{\mathbf{S}}_{i} \cdot \hat{\mathbf{S}}_{i+x}-\hat{\mathbf{S}}_{i} \cdot \hat{\mathbf{S}}_{i+y}\right)
$$

where $x=(1,0), y=(0,1)$. As shown in Fig. 17, the exact diagonalization results for $N=16$ and $N=36$ indicate that the susceptibility associated with this kind of symmetry breaking, $\chi_{\mathrm{C}}$, decreases with the system size. In order to exclude an anomalous size scaling we have extended the calculation up to $N=64$. Our quantum Monte Carlo results, which reproduce quite well the ED data, rule out clearly the columnar dimerization.

The above result is in disagreement with the conclusions of several series expansion studies.64.65 However, as stated in Ref. 65 , the series for $\chi_{\mathrm{C}}$ are very irregular and do not allow a meaningful extrapolation to the exact result. In our calculation instead, even the ED results for $N \leq 36$, are already conclusive.

Having established that the columnar susceptibility is bounded, it is now important to study the response of the $J_{1}-J_{2}$ model to a small field coupled to the perturbation

$$
\hat{O}_{\mathrm{T}}=\sum_{i} e^{i \mathbf{Q}_{0} \cdot \mathbf{r}_{i}} \hat{\mathbf{S}}_{i} \cdot \hat{\mathbf{S}}_{i+x}
$$

with $\mathbf{Q}_{\mathbf{0}}=(\pi, 0)$, explicitly breaking the translation invariance of the Hamiltonian. The evaluation of $\chi_{\mathrm{T}}$, with a reasonable accuracy, is a much more difficult task. In fact in this case the ED values of the susceptibility for $N=16$ and $N=32$ increase with the size and much more effort is then required to distinguish if this behavior corresponds to a spontaneous symmetry breaking in the thermodynamic limit. As 

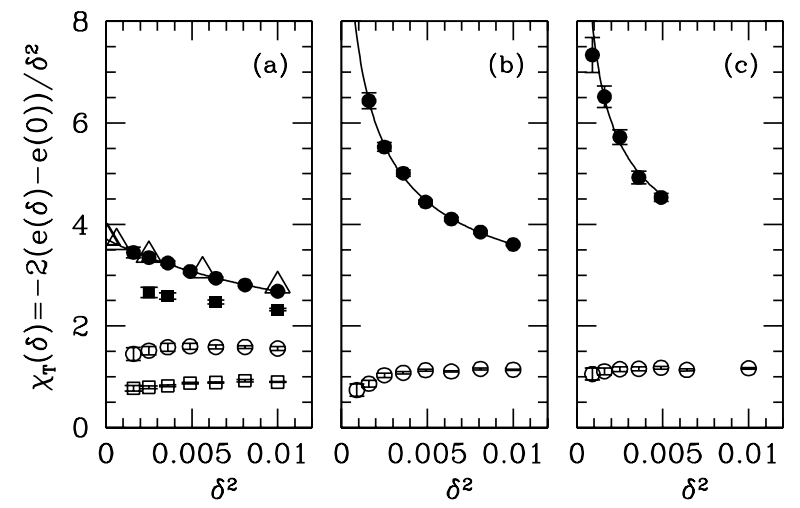

Figure 18: $\quad \chi_{\mathrm{T}}(\delta)$ associated to $\hat{O}_{\mathrm{T}}$ for $J_{2} / J_{1}=0.5, N=32(\mathrm{a}), N=64$ (b) and $N=100$ (c): FN (empty squares), GFMCSR (full squares), FN with LS (empty circles), GFMCSR with LS (full circles), exact (empty triangles).

it is shown in Fig. 18 (a), the FN technique, starting from a guiding wavefunction without dimer order, is not able to reproduce the actual response of the system to $\hat{O}_{\mathrm{T}}$, even on small sizes. The GFMCSR technique allows us to get an estimate of the susceptibility which is a factor of three more accurate, but not satisfactory enough. In order to improve on this estimate, we have attempted to include in the SR conditions many other, reasonably simple, correlation functions (such as the spin-spin correlation functions $\hat{\mathbf{S}}_{i} \cdot \hat{\mathbf{S}}_{j}$ for $\left|r_{i}-r_{j}\right|>\sqrt{2}$ ), but without obtaining a sizable change of the estimate of $\chi_{\mathrm{T}}$. In fact, the most effective SR conditions are those obtained with operators more directly related to the Hamiltonian. 18.19

After many unsuccessful attempts, we have realized that it is much simpler and straightforward to improve the accuracy of the guiding wavefunction itself. This can be obtained by applying a generalized Lanczos operator $(1+\alpha \hat{\mathcal{H}})$ to the variational wavefunction $\left|\psi_{G}\right\rangle$, where $\alpha$ is a variational parameter. This defines the so-called one Lanczos step (LS) wavefunction. 76 In the present model by using the LS wavefunction, a clear improvement (by about a factor of 3) on the variational estimate of the ground-state energy is obtained at all strengths of frustration (see Tab. 2). With this starting point the GFMCSR provides an estimate of the ground-state energy which is basically exact for moderate frustration and remarkable accurate for $J_{2} / J_{1}=0.4$ and 0.5. More importantly, as shown in Fig. 18 (a), the LS wavefunction allows a much better estimate of the susceptibility. This calculation was obtained by including in the SR conditions the energy, the spin-spin correlation functions up to next-nearest-neighbors, distinguishing also $\hat{S}_{i}^{z} \hat{S}_{j}^{z}$ and $\left(\hat{S}_{i}^{x} \hat{S}_{j}^{x}+\hat{S}_{i}^{y} \hat{S}_{j}^{y}\right)(p=4)$. The mixed averages of these correlation functions can be computed over both the wavefunction $\left|\psi_{G}\right\rangle$ and the LS wavefunction $(1+\alpha \hat{\mathcal{H}})\left|\psi_{G}\right\rangle$ during the same Monte Carlo simulation. Thus with a LS wavefunction one can also easily double the number of constraints that are effective to improve the accuracy of the method $(p=8)$. 
In this case, we have tested that it is irrelevant to add further long-range correlation functions in the SR conditions even for large size.

By increasing the size [see Figs. 18], the response of the system is very strongly enhanced, in very close analogy with the one dimensional model in the dimerized phase [see Fig. 16 (b)]. This is obtained only with the GFMCSR technique, since as shown in Fig. 18, the combination of FN and Lanczos step alone, is not capable of detecting these strongly enhanced correlations. For $N=100$ the GFMCSR increases by more than one order of magnitude the response of the system to the dimerizing field. Of course, for a definite conclusion, one should check whether the susceptibility diverges as the volume squared, as implied by Eq. (27). However, in order to obtain quantitatively reliable zero-field extrapolations (81), the limit of very small fields has to be reached. This is in general possible within exact diagonalization (see Fig. 16) but it is rather difficult within a stochastic technique like the GFMC which is always affected by a statistical error.

\section{Conclusions}

In this paper we have studied the interplay between frustration and zero-point quantum fluctuations in the ground state of the triangular and $J_{1}-J_{2}$ Heisenberg antiferromagnets. These frustrated systems are the simplest examples of twodimensional spin models in which quantum effects may be strong enough to destroy the classical Néel order, thus stabilizing a ground state with symmetries and correlations different from their classical counterparts. For this reason, in the last few years, they have attracted much theoretical interest even if a general consensus on the nature of their ground state has not yet been achieved. With this work, by using several techniques including the Green function Monte Carlo with Stochastic Reconfiguration, 1728 a quantum Monte Carlo method recently developed to keep under control the sign problem, we have put on firmer grounds the conclusions on the ground-state properties of these frustrated models.

Despite the fact that the spin-half Heisenberg antiferromagnet on the triangylar lattice was the first historical candidate for a non-magnetic ground state, 34, 38 all our results point toward the existence of zero-temperature long-range Néel order. In fact, our quantum Monte Carlo simulations provide robust evidences for a gapless spectrum and for a value of the order parameter that, although reduced (by about 59\%) with respect to the classical case, remains finite in the thermodynamic limit. This is partially in agreement with the conclusions of finite-temperature calculations 47 suggesting a ground state with a small but nonzero long-range antiferromagnetic order and with series expansions studies 16 indicating the triangular antiferromagnet to be likely ordered but close to a critical point. However, in our simulation, which to our knowledge represents the first attempt to perform a systematic finite-size scaling analysis, the value of the thermodynamic order parameter is sizeable, indicating the presence of stable long-range order. Moreover, the accuracy of the finite-size spin-wave predictions indicates that the spin-wave theory is a reliable analytical approximation to describe the ground-state properties of the 
present model. In particular, the effectiveness of the spin-wave theory in reproducing on finite sizes the low-energy excitation spectrum provides further support to the existence of long-range Néel order in the ground state, suggesting also that the value of the uniform spin susceptibility should be very close to the spin-wave result. We believe that our results, together with the clear indications recently provided by Bernu and co-workers 13 with a symmetry analysis of the low-energy excitation spectra, finally solve the issue of the ordered nature of the ground state of the Heisenberg antiferromagnet on the triangular lattice.

The effects of quantum fluctuations are more remarkable in the $J_{1}-J_{2}$ Heisenberg model, where the combined effect of frustration and zero-point motion interferes with the mechanism of spontaneously broken symmetry, giving rise to a non-magnetic ground state of purely quantum-mechanical nature. In fact, our spinwave, exact diagonalization, and quantum Monte Carlo results indicate that quantum fluctuations are able to melt the antiferromagnetic long-range order in the regime of strong frustration, driving the ground state into a quantum disordered phase at $J_{2} / J_{1} \simeq 0.4$. In addition, with Lanczos and quantum Monte Carlo calculations we have studied the susceptibilities for the most important crystal symmetry breaking operators. Our results, while casting serious doubt on the conclusions of series expansion studies 65.60 indicate the plaquette RVB, with spontaneously broken translation symmetry and no broken rotation symmetry, as a more plausible ground state in the non-magnetic phase. However further investigation is needed to clarify the nature of the ground state in the non-magnetic phase and a more refined numerical investigation is now in progress 7 . In the ordered phase, instead, similarly to the triangular case, we find a remarkable agreement between the spin-wave low-energy excitation spectrum and the exact and quantum Monte Carlo results. This suggests that the value of the uniform spin susceptibility should be very close to the spin-wave prediction up to $J_{2} / J_{1} \simeq 0.30$.

Our results could be also verified experimentally on the novel realizations of these frustrated models, like the triangular $\mathrm{K} / \mathrm{Si}(111)$ : $\mathrm{B}$ interface, $\mathrm{O}$ and the $\mathrm{Li}_{2} \mathrm{VOSiO}_{4}$, $\mathrm{Li}_{2} \mathrm{VOGeO}_{4}$ compounds $\mathrm{O}$ quite recently argued to be well described by a spinhalf $J_{1}-J_{2}$ Heisenberg model on the square lattice. Forthcoming measurements under pressure could also allow one to tune the $J_{2} / J_{1}$ ratio and to investigate the properties of these systems in various regimes of frustration.

\section{Acknowledgements}

I wish to thank Sandro Sorella for three years of enlightening teachings. It is also a pleasure to acknowledge the stimulating collaboration with Federico Becca, Alberto Parola, Giuseppe Santoro, and Adolfo Trumper. Thanks to Valerio Tognetti, Attilio Rigamonti and Pietro Carretta for suggestions and stimulating discussions, and to Claire Lhuillier for useful advice and for a careful reading of the Ph.D. thesis from which the present work has been adapted. Special thanks to Francesca Ferlaino for discussions, support and warm encouragement.

This work was partially supported by MURST (COFIN99). 


\section{References}

1. H. Bethe, Z. Phys 71, 205 (1931)

2. E. J. Neves and J. F. Perez, Phys. Lett. A 114, 331 (1986).

3. P. W. Anderson, Science 235, 1196 (1987).

4. J. D. Reger and A. P. Young, Phys. Rev. B 37, 5978 (1988).

5. P. Chandra, P. Coleman, and A. I. Larkin, Phys. Rev. Lett. 64, 88 (1990).

6. H. H. Weitering, X. Shi, P. D. Johnson, J. Chen, N. J. DiNardo, and K. Kempa, Phys. Rev. Lett. 78, 1331 (1997).

7. C. S. Hellberg and S. C. Erwin, Phys. Rev. Lett. 83, 1003 (1999).

8. R. Melzi, P. Carretta, A. Lascialfari, M. Mambrini, M. Troyer, P. Millet, and F. Mila, Phys. Rev. Lett. 85, 1318 (2000); R. Melzi, S. Aldrovandi, F. Tedoldi, P. Carretta, P. Millet, F. Mila, e.print cond-mat/0101066.

9. P. Carretta, private communication.

10. Q. F. Zhong and S. Sorella, Europhys. Lett. 21, 629 (1993).

11. P. W. Anderson, Phys. Rev. 86, 694 (1952).

12. E. Dagotto, Rev. Mod. Phys. 66, 763 (1994).

13. B. Bernu, C. Lhuillier, and L. Pierre, Phys. Rev. Lett. 69, 2590 (1992); B. Bernu, P. Lecheminant, C. Lhuillier, L. Pierre, Phys. Rev. B 50, 10048 (1994).

14. E. Manousakis, Rev. Mod. Phys. 63, 1 (1991).

15. N. Trivedi and D. M. Ceperley, Phys. Rev. B 41, 4552 (1990).

16. H. J. M. van Bemmel, D. F. B. ten Haaf, W. van Saarloos, J. M. J. van Leeuwen, and G. An, Phys. Rev. Lett. 72, 2442 (1994); D. F. B. ten Haaf, H. J. M. van Bemmel, J. M. J. van Leeuwen, W. van Saarloos, and D. M. Ceperley, Phys. Rev. B 51, 13039 (1995).

17. S. Sorella, Phys. Rev. Lett. 80, 4558 (1998);

18. S. Sorella and L. Capriotti, Phys. Rev. B 61, 2599 (2000).

19. L. Capriotti, A. E. Trumper, and S. Sorella, Phys. Rev. Lett. 82, 3899 (1999).

20. L. Capriotti and S. Sorella, Phys. Rev. Lett. 84, 3173 (2000).

21. E. Lieb and D. Mattis, J. Math. Phys. 3, 749 (1962).

22. W. Marshall, Proc. R. Soc. London Ser. A 232, 48 (1955).

23. R. A. Horn and C. R. Johnson, Matrix Analisys (Cambridge University Press, 1985).

24. J. Richter, N. B. Ivanov, and K. Retzlaff, Europhys. Lett. 25, 545 (1994).

25. P. Azaria, B. Delamotte and D. Mouhanna, Phys. Rev. Lett. 70, 2483 (1993).

26. H. J. Scultz and T. A. L. Ziman, Europhys. Lett. 18, 355 (1992); H. J. Scultz, T. A. L. Ziman, and D. Poilblanc, J. Phys. I (France) 6, 675 (1996).

27. P. W. Anderson, in Basic Notions of Condendes Matter Physics (The Benjamin/Cummings Publishing Company, Menlo Park, CA, 1984).

28. H. Neurberger and T. Ziman, Phys. Rev. B 39, 2608 (1989).

29. D. S. Fisher, Phys. Rev. B 39, 11783 (1989).

30. P. Hasenfrantz and F. Niebermayer, Z. Phys. B 92, 91 (1993).

31. L. Capriotti, Ph.D. Thesis, SISSA, Trieste, 2000.

32. C. Lavalle, S. Sorella and A. Parola, Phys. Rev. Lett. 80, 1746 (1998).

33. S. Chakravarty, B. I. Halperin, and D. Nelson, Phys. Rev. Lett. 60, 1057 (1988).

34. P. W. Anderson, Mater. Res. Bull. 8, 153 (1973).

35. A. Auerbach, Interacting electrons and quantum magnetism (Springer-Verlag, New York, 1994); A. Auerbach, F. Berruto and L. Capriotti, in Field Theories for LowDimensional Condensed Matter Systems edited by G. Morandi, P. Sodano, A. Tagliacozzo, and V. Tognetti (Springer-Verlag, Berlin/Heidelberg, 2000).

36. P. Sindzingre, P. Lecheminant and C. Lhuillier, Phys. Rev. B 50, 3108 (1994).

37. S. Liang, B. Doucot, and P. W. Anderson, Phys. Rev. Lett. 61, 365 (1988); B. Suther- 
land, Phys. Rev. B 37, 3786 (1988).

38. P. Fazekas and P. W. Anderson, Philos. Mag. 30, 423 (1974).

39. T. Oguchi, H. Nishimori, and Y. Taguchi, J. Phys. Soc. Jpn. 55, 323 (1986)

40. H. Nishimori and H. Nakanishi, J. Phys. Soc. Jpn. 57, 626 (1988); 58, 2607 (1989); 58, 3433 (1989).

41. M. Imada, J. Phys. Soc. Jpn. 56, 311 (1987); 58, 2650 (1989).

42. R. Deutscher, H. V. Everts, S. Miyashita, and M. Wintel, J. Phys. A 23, L1043 (1990).

43. P. W. Leung and K. J. Runge, Phys. Rev. B 47, 5861 (1993).

44. V. Kalmeyer and R. B. Laughlin, Phys. Rev. Lett. 59, 2095 (1987).

45. D.A. Huse and V. Elser, Phys. Rev. Lett. 60, 2531 (1988).

46. R. Singh and D. Huse, Phys. Rev. Lett. 68, 1766 (1992).

47. N. Elstner, R. R. P. Singh, and A. P. Young, Phys. Rev. Lett. 71, 1629 (1993).

48. K. Yang, L. K. Warman, and S. M. Girvin, Phys. Rev. Lett. 70, 2641 (1993).

49. M. Boninsegni, Phys. Rev. B 52, 15304 (1995).

50. T. Oguchi, H. Kitatani, and H. Nishimori, J. Phys. Soc. Jpn. 56, 3858 (1987).

51. Th. Jolicoeur and J. C. Le Guillou, Phys. Rev. B 40, 2727 (1989).

52. S. J. Miyake, J. Phys. Soc. Jpn. 61, 983 (1992).

53. A. Chubukov, S. Sachdev, and T. Senthil, J. Phys.: Condens. Matter 6, 8891 (1994).

54. L. O. Manuel, A. E. Trumper and H. A. Ceccatto, Phys. Rev. B 57, 8348 (1998).

55. M. Takahashi, Phys. Rev. B 40, 2494 (1989).

56. J. E. Hirsch and S. Tang, Phys. Rev. B 40, 4769 (1989).

57. A. E. Trumper, L. Capriotti, and S. Sorella, Phys. Rev. B 61, 11529 (2000).

58. F. Franjic and S. Sorella, Prog. Teor. Phys. 97, 399 (1997).

59. L. Capriotti, R. Vaia, A. Cuccoli, and V. Tognetti, Phys. Rev. B 58, 273 (1998); L. Capriotti, A. Cuccoli, V. Tognetti, P. Verrucchi, and R. Vaia, Phys. Rev. B 60, 7299 (1999).

60. E. Dagotto and A. Moreo, Phys. Rev. Lett. 63, 2148 (1989).

61. P. Chandra and B. Doucot, Phys. Rev. B 38, 9335 (1988).

62. H. A. Ceccatto, C. J. Gazza, and A. E. Trumper, Phys. Rev. B 45, 7832 (1992).

63. A. E. Feiguin, C. J. Gazza, A. E. Trumper, and H. A. Ceccatto, Phys. Rev. B 52, 15 043 (1995).

64. M. P. Gelfand, R. R. P. Singh, and D. A. Huse, Phys. Rev. B 40, 10801 (1989).

65. R. R. P. Singh, Z. Weihong, C. J. Hamer, and J. Oitmaa, Phys. Rev. B 60, 7278 (1999).

66. M. Zithomirski and K. Ueda, Phys. Rev. B 54, 9007 (1996).

67. N. Read and S. Sachdev, Phys. Rev. Lett. 66, 1773 (1991); S. Sachdev and N. Read, Int. J. Mod. Phys. B 5, 219 (1991).

68. F. Figueirido, A. Karlhede, S. Kivelson, S. Sondhi, M. Rocek, and D. S. Rokhsar, Phys. Rev. B 41, 4619 (1989).

69. M. Calandra and S. Sorella, Phys. Rev. B 57, 11446 (1998).

70. V. N. Kotov, J. Oitmaa, O. P. Sushkov, and Z. Weihong, Phys. Rev. B 60, 14613 (1999).

71. M. S. L. du Croo de Jongh, J. M. J. van Leeuween and W. van Saarloos, Phys. Rev. B 62, 14844 (2000).

72. G. Santoro, S. Sorella, L. Guidoni, A. Parola, and E. Tosatti, Phys. Rev. Lett. 83, 3065 (1999).

73. F. D. M. Haldane, Phys. Rev. B 25, 4925 (1982); 26, 5257 (1982).

74. G. Castilla, S. Chakravarty, and V. J. Emery, Phys. Rev. Lett. 75, 1823 (1995).

75. S. Eggert, Phys. Rev. B 54, 9612 (1996).

76. E. S. Heeb, and T. M. Rice, Europhys. Lett. 27, 673 (1994); E. S. Heeb, Ph.D. Thesis, ETH, Zürich, 1994.

77. L. Capriotti, F. Becca, A. Parola, and S. Sorella, in preparation. 